\title{
The Vulnerability of Price Stabilization Schemes to Speculative Attack
}

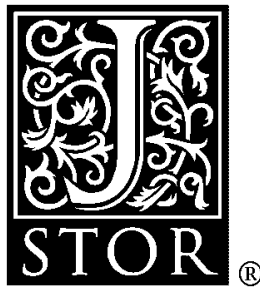

Stephen W. Salant

The Journal of Political Economy, Vol. 91, No. 1. (Feb., 1983), pp. 1-38.

Stable URL:

http://links.jstor.org/sici?sici=0022-3808\%28198302\%2991\%3A1\%3C1\%3ATVOPSS\%3E2.0.CO\%3B2-Q

The Journal of Political Economy is currently published by The University of Chicago Press.

Your use of the JSTOR archive indicates your acceptance of JSTOR's Terms and Conditions of Use, available at

http://www.jstor.org/about/terms.html. JSTOR's Terms and Conditions of Use provides, in part, that unless you have obtained prior permission, you may not download an entire issue of a journal or multiple copies of articles, and you may use content in the JSTOR archive only for your personal, non-commercial use.

Please contact the publisher regarding any further use of this work. Publisher contact information may be obtained at http://www.jstor.org/journals/ucpress.html.

Each copy of any part of a JSTOR transmission must contain the same copyright notice that appears on the screen or printed page of such transmission.

The JSTOR Archive is a trusted digital repository providing for long-term preservation and access to leading academic journals and scholarly literature from around the world. The Archive is supported by libraries, scholarly societies, publishers, and foundations. It is an initiative of JSTOR, a not-for-profit organization with a mission to help the scholarly community take advantage of advances in technology. For more information regarding JSTOR, please contact support@jstor.org. 


\title{
The Vulnerability of Price Stabilization Schemes to Speculative Attack
}

\section{Stephen W. Salant}

\author{
Rand Corporation
}

This paper examines the effects of government attempts to stabilize the prices of commodities by use of buffer stocks. Agricultural goods subject to supply uncertainty as well as depletable resources are considered. In each case, it is shown that the resulting rational expectations competitive equilibrium contains a speculative attack-a situation where the entire government stock is suddenly purchased by previously inactive speculators. The analysis is applied to the historical attempt to peg the gold price, which caused the attack of 1968. The insights gained and the methodology developed also apply to the various international agreements to impose bans on commodity prices which have been proposed by the United Nations Conference on Trade and Development.

Commodity agreements of one kind and another have ... over a half century of experience behind them, all of it a history of failure ... faith [in them] rests either on ignorance of past history or the obstinate belief that what went wrong last time was attributable to lack of will or cleverness, or unwillingness to commit sufficient financial resources to the enterprise, but never to inherent difficulties that could be understood in terms of elementary economic analysis. [HARRY JOHNSON 1977]

\section{Introduction}

Previous attempts to stabilize the prices of commodities by purchases and sales from buffer stocks have ended in failure. In the past buffer

I wish to acknowledge useful discussions on attacks with Katherine Blair, John Bryant, Dale Henderson, Pauline Ippolito, Val Koromzay, Stephen LeRoy, Walter Salant, Steven Salop, T. N. Srinivasan, Sheldon Switzer, Hal Varian, and Peter Zadrozny. I gratefully acknowledge technical assistance from Milton Harris, David Levine, Robert Lucas, and Robert Townsend. 
stocks of gold, silver, and tin were each attacked by speculators anticipating price increases. ${ }^{1}$ Yet similar stabilization schemes continue to be proposed and, in some cases, adopted. For example, in 1975, the secretary general of the United Nations Conference on Trade and Development (UNCTAD) proposed an "Integrated Program for Commodities" to stabilize the prices of 17 commodities by means of purchases and sales from individual buffer stocks. It was proposed that the prices of the following commodities be kept within bands: cocoa, coffee, tea, sugar, hard fibers, jute, cotton, rubber, copper, tin, bananas, wheat, rice, meat, wool, iron ore, and bauxite. Disagreements about the size of the stockpiles resulted in 1979 in a breakdown of the international negotiations. But new proposals are to be expected. At the moment, buffer stocks are being operated to stabilize the prices of tin, natural rubber, and cocoa. Meanwhile, a presidential commission contemplates returning to an international monetary system where the nominal price of gold would be pegged by purchases and sales from gold stocks. Proponents of such schemes either ignore the historical record or feel confident the mistakes of the past can be avoided simply by creating larger buffer stocks in the future. ${ }^{2}$

Such confidence rests on shaky foundations. Although many of the recent agreements and proposals have been evaluated favorably, the methodology underlying these evaluations renders their favorable conclusions worthless. The consequences of a particular commodity agreement cannot be forecast without taking proper account of how it will affect the price expectations of speculators and hence their behavior. Yet previous econometric analyses have ignored completely the impact of such schemes on speculative demand. ${ }^{3}$ This is no small

${ }^{1}$ The government's desperate attempts to stabilize silver prices are summarized in Burke and Levy (1969). The futile attempts to keep a lid on tin prices are summarized in Fox (1974).

${ }^{2}$ See, e.g., Bergsten (1976, p. 9; 1977, p. 9).

${ }^{3}$ In Goreux's evaliation of bands (1978), he assumes away private speculators altogether, although he stresses the desirability of including them and "their reaction to the bufferstock intervention rule" in future work. Two econometric studies include private speculators but fail to capture their reaction to the policy regime facing them. In one study prepared jointly for the departments of State, Treasury, and Commerce by the Commodity Research Unit (CRU) (1977), an econometric model of the copper industry was estimated under the conventional (but erroneous) assumption that agents react only to current and lagged prices. Then-under the (false) assumption that behavior would not be affected by the operation of the government buffer stock-a price forecast was generated. In many of the simulations reported, the stockpile was exhausted during the 15-year simulation period and an upward jump in price resulted. Such price paths are disequilibria and, as such, have no legitimacy as forecasts. No properly specified model would generate them. In another study, Schink and Smith (1976) use the Wharton EFA model to evaluate how the tin market would have functioned with and without a commodity agreement-implicitly assuming speculative behavior would not be affected by the change in regime. Both the CRU study and the article by Schink and Smith are typical of the kind of econometric policy evaluation which is the subject of Lucas's (1976) critique. 
omission since it has been speculators who have thwarted previous attempts at price stabilization.

Some have argued in defense of these econometric policy evaluations that the particular models chosen for the analyses have demonstrated impressive forecasting records in the past. But such records are irrelevant to the ability of these models to forecast the consequences of a new policy that will almost surely change the buy-sell rules of speculators. Lucas (1976) made an analogous point several years ago in his critique of the practice of using the econometric models then in existence to evaluate alternative macroeconomic policy proposals. He concluded that "comparisons of the effects of alternative policy rules using current macro-econometric models are invalid regardless of the performance of these models over the sample period or in ex ante short-term forecasting" (p. 141; my emphasis).

This paper develops a methodology in which the consequences of buffer stock proposals can be evaluated properly. The approach is to examine the competitive equilibrium under rational expectations when the government adheres to a specified policy rule. For analytic simplicity, it is assumed that the price stabilization policy attempted by the government is a peg. This policy is shown under plausible assumptions to induce speculative attacks. However, the methodology developed and the insights gained both about speculative attacks and about one-sided bets against breaches of the ceiling price apply equally to policy regimes where ceiling and floor prices differ. ${ }^{4}$ Hence, the methodology developed can be used to analyze the various commodity agreements which have been proposed by UNCTAD.

In Section II, a pegging policy is introduced into the Hotelling model of pure depletion under certainty. The resulting equilibrium is shown to contain a speculative attack - a situation where previously inactive speculators suddenly purchase the remaining government stock. Speculative attacks are popularly regarded as the product of either irrationality or unfounded expectations about future prices. By proving that attacks must occur even when agents behave optimally and have rational expectations about future prices, it is shown that psychological factors are not needed to explain their occurrence. An attack occurs when total stocks fall below an endogenously determined threshold-as they eventually must with a nonrenewable resource because of continual depletion. An earlier version of this certainty case, discussed briefly in Salant and Henderson (1978), has been adapted by Koromzay (1978) and Krugman (1979) subsequently

\footnotetext{
${ }^{4}$ A general computerized version of the model could be used first to analyze specific proposals (be they bands, pegs, ceilings, or floors) and later to forecast prices once a proposal is implemented. An excellent start on such a model has been developed independently by Gardner (1979). The merit of the present analytical treatment is its insights into the properties of such a model.
} 
to explain attacks on fixed exchange rate regimes, by Flood and Garber (1981) to study the collapse of a banking system, by Garber (1981) to model runs on savings and loan institutions, and by Flood and Garber (1982) to study the sudden monetary contraction which would result if an attack terminated a government's attempt to monetize gold.

In Section III, the model is generalized to incorporate uncertainty. Random additions, interpreted as new harvests, are assumed to augment each period the remaining private stock of an agricultural good. It has been shown by Townsend (1977) under related circumstances that the government cannot successfully peg the price forever by operating a buffer stock; but he did not go on to investigate the stochastic equilibrium which does result when such a futile policy is attempted. Such an analysis is conducted in Section III, where a pegging policy is introduced into the standard agricultural carry-over model of Gustafson (1958), Samuelson (1971), and Kohn (1978). This model is a generalization of the Hotelling model of Section II. As in Section II, a speculative attack is shown to occur in equilibrium whenever total stocks fall below an endogenous threshold. But now the possibility exists that random additions will keep the total stock above the threshold. The probability of this event depends on the type of policy pursued and its underlying parameters. If the policy is attempted pegging and the official price is set so low that it induces more consumption than the mean harvest, then a speculative attack occurs with probability one. ${ }^{5}$ Indeed, the certainty case of Section II can be regarded as a degenerate example of this proposition where the harvest density collapses to a mass point at zero. A more revealing case is when the consumption induced by the official price just matches the expected harvest. Then, on average, no depletion of the government stock will occur and it seems natural to think that the attack threshold will never be crossed-at least if the initial government stockpile is sufficiently large. This turns out to be true only if harvests do not fluctuate at all. Otherwise, an attack is virtually inevitable. Indeed, as Wenocur and Salant (1981) have established, such attacks almost surely recur infinitely often-regardless of the initial size of the government stockpile. Section IV discusses the economic function of speculative attacks and concludes the paper.

${ }^{5}$ If the commodity is a pure depletable, any buffer stock used to enforce a ceiling on its price (e.g., a band) will induce an attack. If the commodity can be replenished stochastically, the probability of an attack depends on the parameters of the stabilization scheme. For example, if the band width is infinite (laissez-faire) no attack will occur; if its width is zero (pegging) - and if $D(\bar{P}) \geqslant E(H)$-an attack will occur with probability one. For any specific proposal a computerized version of the model would permit calculation of all probabilities of interest. 


\section{Pure Depletion under Certainty}

In this section, we introduce a government policy of price pegging into the standard Hotelling model of an exhaustible resource. Since this model contains neither randomness nor additions to stocks, it permits attention to be focused exclusively on the causes of speculative attacks.

\section{A. Equilibrium in the Hotelling Model with No Government Intervention}

We begin by summarizing the simplest laissez-faire Hotelling model in discrete time. ${ }^{6}$ Readers unfamiliar with this model and wishing to see a rationalization of its assumptions within a general equilibrium framework are directed to Appendix A.

There are two types of agents: consumers and extractor-speculators. The aggregate behavior of consumers of the exhaustible resource is summarized by a demand curve that is time separable, stationary, dependent on the contemporaneous price of the resource and on no other prices, and independent of income. For simplicity, it is assumed that the demand curve intersects the vertical axis at some "choke price," $P^{c}$. Denoting the demand curve by $D\left(P_{t}\right)$, we have: $D\left(P_{t}\right)>0$ for $P_{t}<P^{c}, D\left(P_{t}\right)=0$ for $P_{t} \geqslant P^{c}$, and $D^{\prime}\left(P_{t}\right)<0$ for $P_{t}<P^{c}$.

Extractor-speculators are assumed to have perfect foresight, a finite initial stock of the resource, zero extraction costs, and zero costs of storage. Denote the exogenous stationary real rate of interest by $r$, the price sequence which extractor-speculators take as given by $\left\{P_{t}\right\}$, their aggregate net sales (or purchases, if negative) by $x_{t}$, and the stock remaining at $t$ prior to net sales then by $K_{t}$. Extractor-speculators are assumed to maximize their wealth: that is, maximize

$$
\sum_{t=1}^{\infty} \beta^{t-1} P_{t} x_{t}
$$

subject to

$$
\begin{aligned}
K_{1} & =\bar{K}, \\
K_{t+1} & =K_{t}-x_{t}, \\
K_{t} & \geqslant 0, \text { for } t=1,2, \ldots,
\end{aligned}
$$

where $\beta=1 /(1+r)$ and $\left\{P_{t}\right\}$ is given parametrically. In Section II $B$, the forces which motivate a speculative attack are highlighted by provisionally assuming that speculation (purchases from the market by

\footnotetext{
${ }^{6}$ For a more complete exposition of this model, see secs. 2 and 3 of Weinstein and Zeckhauser (1975).
} 
extractor-speculators for resale in the future) cannot occur. Hence, in that subsection only, we impose the constraint that $x_{t} \geqslant 0$ in the foregoing maximization problem.

Since the objective function of the extractor-speculators is linear in $x_{t}$, sales will take place only in periods where the discounted price is highest. For the same reason, speculative purchases will take place only in those periods where the discounted price is lowest. In equilibrium, the price must increase in each period by no more than the rate of interest. For suppose $P_{t+1}>P_{t}(1+r)$ on some $t$. Then the extractor-speculators could make infinite profits by purchasing an infinite amount of the resource in period $t$, storing it, and then reselling it at $t+1$. Since there exists only a finite amount of the resource, such behavior would create excess demand at $t$ and could not occur in equilibrium. We now define and characterize more fully a laissezfaire, competitive equilibrium in the Hotelling model.

A competitive equilibrium is a price sequence $\left\{P_{t}\right\}$ and a net sales sequence $\left\{x_{t}\right\}$ such that no other net sales sequence can strictly increase the discounted wealth of the extractor-speculators at the given prices and such that $\left\{x_{t}\right\}$ satisfies the demand induced each period by the price sequence $\left\{P_{t}\right\}$. Hotelling established that a unique competitive equilibrium exists under laissez-faire, which can be characterized as follows:

$$
\begin{gathered}
P_{t}=P_{1}(1+r)^{t-1}, \\
\sum_{t=1}^{\infty} D\left[P_{1}(1+r)^{t-1}\right]=\bar{K},
\end{gathered}
$$

and

$$
x_{t}=D\left(P_{t}\right), \text { for } t=1,2, \ldots
$$

In equilibrium, the price of the resource rises at the real rate of interest from that unique level which induces the sum of the demands over time to equal the initial stock $(\bar{K})$.

\section{B. Competitive Equilibrium with Government Pegging but with No Speculative Purchases}

We now consider how the equilibrium would be displaced if the government intervened. Specifically, suppose the government attempted to peg the real price of the resource (call it "oil") by standing ready to purchase whatever oil is offered or to sell, if necessary, all its limited stock to maintain the official price relative to that of some storable background good. ${ }^{7}$ Denote by $G_{t}$ the government stock of oil at the beginning of period $t$, prior to government intervention in that pe-

${ }^{7}$ See model 2 of App. A. 
riod. Then the government policy of attempting to peg the market price at the official rate $\bar{P}$ may be described as follows: If $P_{t}>\bar{P}, G_{t+1}$ $=0$; if $P_{t}=\bar{P}, G_{t+1} \in[0, C]$; and if $P_{t}<\bar{P}, G_{t+1}=C$, where $C$ is the exogenous maximum stock of oil the government can hold due to physical or financial constraints. That is, if the market price ever exceeds the official level, it is because the buffer stock manager has run out of reserves to sell. If the market price ever drops below the official level, it is because the manager cannot acquire additional reserves. For simplicity, it is assumed (until the generalization in Sec. III) that $C$ is so large that the market price never falls below $\bar{P}$. Finally, it is assumed that the government has no oil initially $\left(G_{1}=0\right)$ but has a stock of background good large enough to buy the entire private reserve of oil $(\bar{K})$ at the official price.

If the official price is set below the price which would prevail under laissez-faire, the laissez-faire equilibrium will persist, since no one would sell oil to the government at a loss and the government by assumption has no initial stocks of oil to sell. If the official price is set above the laissez-faire price but below the choke price, the government will bid up the market to $\bar{P}$ by its purchases. But it cannot sustain this market price forever. Since reserves acquired by the government are finite, they cannot satisfy the demand induced by the official price forever-even if the government initially purchased every drop of oil on the planet.

The market incentives which trigger an attack can best be understood by considering initially the equilibrium price sequence which the government policy would induce in the absence of speculative purchases. That is, we provisionally require that $x_{t} \geqslant 0$ and compute the competitive equilibrium with attempted price pegging. Since (by assumption) the government follows its policy rule, it would prevent the price from falling below the official level $(\bar{P})$ by purchasing at that rate of exchange all the oil which extractors offered and would allow the price to rise above that level only if it ran out of reserves to sell. Since (by assumption) the extractors maximize the sum of discounted profits, they would sell their stock $(\bar{K})$ at the highest discounted price (if the highest discounted price occurred in different periods, there would be indifference as to the allocation of the stock among such periods). In equilibrium, the extractors must voluntarily sell oil to the government in the first period and, in the absence of speculation, directly to consumers after the buffer stock is exhausted. Thus, the equilibrium price path must consist of two discontinuous pieces and can be constructed from the curves in figure 1 . The first piece of the path is horizontal at the official price. ${ }^{8}$ The second, higher piece rises

${ }^{8}$ The diagram and description in the text are correct for continuous time. In discrete time, a bothersome integer problem arises. In each period of government sales except 


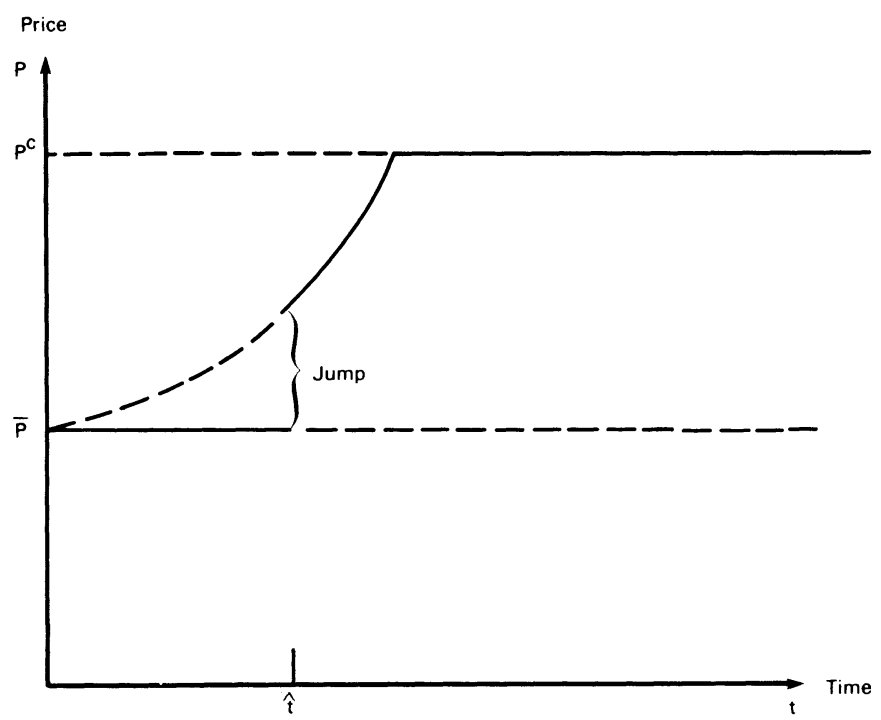

FIG. 1.-The equilibrium price path with no speculative purchases

at the rate of interest from a level which has a present value equal to $\bar{P}$ until the choke price $\left(P^{c}\right)$ is reached. Given such a price path, extractors would be willing to sell either in the first period or between the time the price jumps up and the time it reaches the choke price. Define $\hat{t}$ as the last period before the upward jump. If $\hat{t}=1$, the price would initially be $\bar{P}$ but then would rise at the rate of interest. Since $\bar{P}$ is assumed to exceed the laissez-faire price, however, such a path would induce less cumulative demand than is required to exhaust the initial stock $(\bar{K})$. Hence, any equilibrium must have $\hat{t} \geqslant 2$. The larger $\hat{t}$, the greater the cumulative demand which is induced by the resulting price path. If, for example, $\hat{t}$ is increased from $k$ to $k+1$, cumulative demand would increase by $D(\bar{P})-D\left[\bar{P}(1+r)^{k}\right]$. By lengthening $\hat{t}$ sufficiently, we can construct a path which induces a cumulative demand of $\bar{K}$. In this equilibrium, the price will jump up at $\hat{t}+1 .{ }^{9}$ Two

the iast, the market price equals $\bar{P}$. In the final period of government sales, however, the stock sold at the official price may be smaller than $D(\bar{P})$ and hence the market price can exceed $\bar{P}$ (see n. 9).

${ }^{9}$ In continuous time, the jump occurs at $\hat{t}$, where $\hat{t}$ solves the following: $\hat{t} D(\bar{P})+\int_{t=\hat{t}}^{\infty}$ $D\left(\bar{P} e^{r t}\right) d t=\bar{K}$. In discrete time, $\hat{t}$ is the final period of government sales. In the light of n. $8, \hat{t}$ is that integer which solves the following equation:

$$
(\hat{t}-1) D(\bar{P})+\sum_{t=\hat{t}+1}^{\infty} D\left[\bar{P}(1+r)^{t-1}\right]+D\left(P_{\hat{t}}\right)=\bar{K}
$$

for some $P_{\hat{t}}$ such that $\bar{P}(1+r)^{\hat{i}-1}>P_{\hat{t}} \geqslant \bar{P}$. 
cases might arise. In the first, $\bar{P}(1+r)^{\hat{t}}<P^{c}$; in this case the price jumps to a level which induces positive demand. In the second, $\bar{P}(1+$ $r)^{\hat{t}} \geqslant P^{c}$; in this case the price jumps to a level which induces zero demand. In the former case, extractors sell part of their reserves initially and retain the remainder for sale after the price jump. In the latter case, extractors sell all of their reserves initially. In either case, however, the price must jump up at $\hat{t}+1$ in any equilibrium.

\section{Competitive Equilibrium with Government Pegging Once Speculative Purchases Are Permitted}

It has been shown that the equilibrium price path in the absence of speculative purchases always has an upward jump. We now consider how the equilibrium changes when such purchases are permitted. That is, we drop the nonnegativity constraint on $x_{t}$. The old equilibrium price path would then give extractor-speculators an incentive to buy infinite amounts at the official price in the period before the jump $(\hat{t})$ and to sell sometime afterward. ${ }^{10}$ Given any amount to be acquired, the least costly acquisition strategy is to purchase in the last period before the jump-rather than more gradually_since gradual acquisition at the official price would result in unnecessary interest costs.

Infinite speculative purchases cannot occur in equilibrium. If they were attempted, they would cause the buffer stock to be depleted sooner; however, the corresponding subsequent sales would cause the second piece of the price path to begin at a lower level. Indeed, if speculators acquired a large enough stock, the upward jump in the price path would be eliminated altogether and no incentive for a larger acquisition would remain. ${ }^{11}$ The absence of a price jump would induce extractors to sell their entire stock to the government in the first period. It will be shown that such a situation constitutes the new equilibrium.

${ }^{10}$ In continuous time, any upward jump in the price path would induce speculators to make infinite demands the instant before the jump. In discrete time, an upward jump would create the same incentives provided $\bar{P}(1+r)<P^{c}$. If, instead, the official price were set just under the choke price $P^{c}>\bar{P} \geqslant P^{c} /(1+r)$ there would be no incentive for speculators to attack. We assume that $\bar{P}(1+r)<P^{c}$ and omit consideration of the alternative case, which has no counterpart in continuous time. A parallel assumption is made in Sec. III (see n. 21 ).

${ }^{11}$ In continuous time the market price equals $\bar{P}$ until the attack occurs and then rises without any jump at the rate of interest. In discrete time, the market price can lie in the interval $[\bar{P}, \bar{P}(1+r))$ in the period of attack as a result of an integer problem arising from period analysis. Hence there would be no incentive to acquire stocks a period sooner. But those fortunate enough to buy from the government at the official price in the period of the attack would make an immediate gain proportional to their acquisitions. 


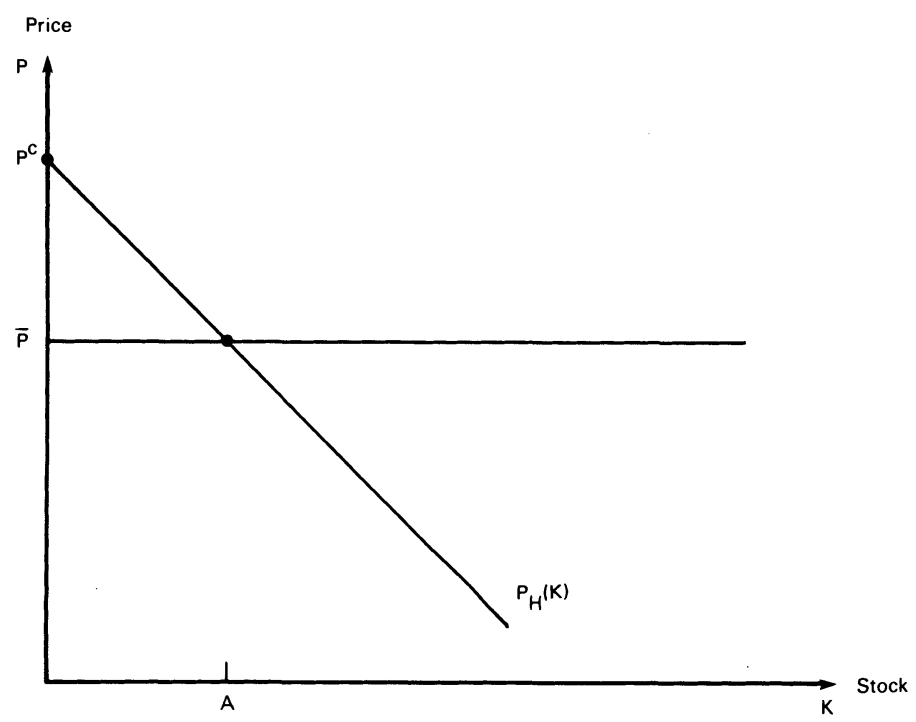

FIG. 2.-Determination of the attack threshold

If speculators acquired some stock $K$ in the attack, the price would (in accordance with the Hotelling principle [1931]) immediately adjust to a level $\left(P_{H}\right)$ permitting absorption of $K$ along a path rising over time at the rate of interest. The larger the stock to be absorbed, the lower the initial price on the second piece of the price path: $P=$ $P_{H}(K), P_{H}^{\prime}(K)<0$. In the simple case under consideration, this function can be written implicitly as a single equation:

$$
\sum_{t=1}^{\infty} D\left[P_{H} \cdot(1+r)^{t-1}\right]=K .
$$

Let $A$ denote the smallest government stock consistent with maintenance of the official price. In the period when the government stock first declines below $A$, speculators will suddenly buy the entire remaining government stockpile at $\bar{P} ; A$ is referred to as the "attack threshold." Analytically, it is defined by $P_{H}(A)=\bar{P}$; graphically, it is determined in figure 2.

It is easy to verify that the attack could occur neither earlier nor later. If the attack were to occur earlier, when the government stock exceeded $A$, speculators would take a loss. For the market price in the period of the attack would then fall beneath $\bar{P}$. Since it is not optimal for foresighted speculators to purchase in anticipation of a loss, an attack on a stock larger than $A$ is a disequilibrium. Alternatively, suppose one period had elapsed with government reserves less than $A$ before the attack occurred. Then the stocks acquired would be smaller than $A-D(P)$. But then the market price in the period of the 
attack would exceed $\bar{P}(1+r)$ and in anticipation of that price there would have been infinite demand by speculators in the previous period at the official price.

In the equilibrium, the government maintains the official price prior to the attack. To do so, the government initially must purchase the offerings of extractors who sell their entire stock at the highest discounted price (the initial price). ${ }^{12}$ The government must then sell to the market at the rate $D(\bar{P})$. When the stock falls below $A$, speculators suddenly attack the remaining reserves; they then sell over time at prices rising without a jump from the official price by the rate of interest. ${ }^{13}$ This scenario is an equilibrium since (1) the actions of the extractor-speculators, consumers, and the buffer stock manager are compatible (markets clear); (2) the government is following its policy rule; and (3) the extractor-speculators cannot increase discounted profits at the given prices by alternative behavior.

We conclude this section by making two observations. First, this simple model predicts speculators will attack swiftly, with no prior activity, and before consumers have depleted the government buffer stock. Such behavior, which is often regarded as bizarre, has been shown to be the entirely rational response to the market incentives generated by the price-stabilization program. Second, introduction of the buffer stock program raises the initial price and therefore increases the wealth of the extractors relative to laissez-faire. In such a circumstance, it is not surprising that the program has its political adherents. $^{14}$

\section{Generalization to Incorporate Stochastic Replenishment}

\section{A. Introduction}

In the previous section, we introduced a government pegging policy into the simplest Hotelling model of an exhaustible resource. Since

\footnotetext{
${ }^{12}$ That extractors sell their entire reserves initially is a consequence of the assumption that marginal extraction costs are constant. Readers finding the implication unrealistic should consider the case where marginal extraction costs are an increasing function of the rate of extraction. This case also generates a speculative attack but is not considered in the text because it is slightly more complicated.

${ }^{13}$ The description in the text is correct in continuous time: i.e., government reserves decline to $A$, the attack threshold defined by $\int_{X=0}^{\infty} D\left(\bar{P} e^{m}\right) d x=A$. An attack then occurs and the market price at the instant of attack is $\bar{P}$. In discrete time, a bothersome integer problem arises. As a result, the market price in the period of the attack can lie in the interval $[\bar{P}, \bar{P}(1+r))$. Since this is an artifact of period analysis, we do not label this a price jump.

${ }^{14}$ In reality, the government must first acquire the background good (financing) needed to swap for the oil. The claim in the text is true if none of this burden is imposed on the extractors. Otherwise, they too might lose even though their profits increase from sales of the resource.
} 
there are no additions to aggregate stocks in that model, the reserves of the government decline steadily-permitting attention to be focused on the incentives for speculative attacks which a reduced government stock creates.

With this preliminary understanding of the determinants of speculative attacks, we are ready to consider whether attacks persist in more complex environments. In particular, we examine the effects of a stabilization policy in the standard agricultural carry-over model analyzed extensively by Gustafson (1958), Samuelson (1971), Kohn (1978), and many others. In this model, private carry-overs are augmented each period by a random exogenous harvest $\left(\tilde{H}_{t}\right)$ drawn independently from a stationary distribution. As in the Hotelling model, there are two kinds of private agents: consumers-whose demand depends only on the contemporaneous price-and producer-speculators-whose sequential decisions maximize their expected wealth. As in the Hotelling model of Section II, costs of production and storage are assumed to be zero. The previous assumption of parametric price taking is generalized to uncertainty by assuming that producerspeculators take as given the current price and the future probability distributions of prices. In this uncertain environment, producerspeculators are assumed to maximize sequentially their current expectation of future discounted profits. Hence, the laissez-faire Hotelling model of the last section can be regarded as a degenerate case of this more general model where the stationary distribution of random harvests collapses to a mass point at zero.

The laissez-faire equilibrium to this model has been studied repeatedly. Our goal will instead be to deduce the properties of its equilibrium when the government intervenes in an attempt to stabilize the price of a commodity by purchases and sales from a buffer stock. In the text, only a policy of price pegging will be considered. However, as will be apparent, the approach can also be used to evaluate price bands.

In uncertainty models, it is convenient to express all endogenous variables as functions of the state of the system. In this model, the sizes of private and government stocks are the state variables. Since we are considering a stationary infinite-horizon model, the market price in any period can be expressed as a stationary function of the state variables, $P(K, G)$, where $K$ denotes the private stock at the beginning of the period (including the contemporaneous harvest) prior to private sales or purchases, and $G$ denotes the government stock then prior to intervention.

It will be convenient to rewrite the definition of the policy of price pegging in terms of the government stocks $(R)$ at the end of the 
period. Hence the government pegging policy can be expressed as follows:

$$
\begin{gathered}
\text { if } P>\bar{P}, R=0, \\
\text { if } P=\bar{P}, R \in[0, C], \\
\text { if } P<\bar{P}, R=C .
\end{gathered}
$$

We denote this correspondence, which characterizes the government policy, as $R(P)$.

The economy evolves according to the following system of stochastic difference equations:

$$
\begin{gathered}
P_{t}=P\left(K_{t}, G_{t}\right), \\
K_{1}=\bar{K}, G_{1}=\bar{G}, \\
\tilde{K}_{t+1}=K_{t}-\left[D\left(P_{t}\right)+R\left(P_{t}\right)-G_{t}\right]+\tilde{H}_{t+1}, \\
G_{t+1}=R\left(P_{t}\right), \quad t=1,2, \ldots .
\end{gathered}
$$

The first equation indicates the market equilibrium price in period $t$ for any given values of the state variables at the beginning of period $t$. The second indicates the initial values of the state variables. The third and fourth equations describe subsequent transitions in these state variables. Given $P(K, G)$, these equations describe the stochastic evolution of the economy.

The rub lies in characterizing $P(K, G)$. Samuelson (1971) characterized the equilibrium price function in the laissez-faire case by first specifying a stochastic planning problem which is solved in the competitive equilibrium, then analyzing this optimization problem, and finally examining its associated shadow prices. Unfortunately, since there is no reason to think-in the presence of government intervention-that the market equilibrium maximizes anything we can prespecify, Samuelson's approach cannot readily be extended.

Our approach instead will be to analyze a set of restrictions on the price function which must hold if (1) markets clear in each period, (2) producer-speculators maximize at each stage their expected discounted profits, and (3) the government follows its intervention rule. Any price function satisfying these equilibrium conditions must solve the following functional conditions for each state of the system:

$$
K-\{D[P(K, G)]+R[P(K, G)]-G\} \geqslant 0
$$


and

$$
\begin{gathered}
P(K, G)-\beta E_{H} P(K-\{D[P(K, G)] \\
+R[P(K, G)]-G\}+\tilde{H}, R[P(K, G)]) \geqslant 0,
\end{gathered}
$$

with complementary slackness. The term complementary slackness means that at least one of the two conditions must hold with equality. Since $R(\cdot)$ is defined by (1)-(3), the left-hand side of each inequality will depend only on $P(K, G)$. Any function which satisfies these complementary slackness conditions is said to be an equilibrium price function.

These conditions place restrictions on candidate price functions which must hold in any competitive equilibrium. Their economic meaning will now be clarified. The first condition indicates that private stocks at the beginning of any period less the combined amounts absorbed by private consumers and by the government must not be negative. That is, the carry-overs of producer-speculators must be nonnegative. In expressing actual private and government purchases in terms of each sector's desired purchases $(D[\cdot]$ and $R[\cdot]-G)$, we are assuming that the government is following its policy rule and that the current market clears.

The second functional condition indicates that the current price minus the current expectation of next period's discounted price must not be negative. If it were, a disequilibrium would result since speculators would then attempt to buy infinite stocks today with the intention of storing, selling next period, and making infinite expected profits. The assumption that producer-speculators have rational expectations is reflected in the fact that their current expectation of next period's discounted price is equal to the probability-weighted average of the values which the actual equilibrium price function would take at each of the possible realizations of the state variables. The first variable - the private stock which will be available for marketing next period-is equal to the sum of inventories carried privately and the new harvest. The second variable is the government stock carried out of the current period and into the next period. In expressing this latter stock in terms of the exogenous $R(\cdot)$ correspondence, we are assuming that the government is following its policy rule.

Finally, the complementary slackness condition indicates that if speculators currently expect to take a loss by carrying inventories, they will carry none. And if they carry inventories, no loss is currently expected. These conditions must hold if producer-speculators maximize expected discounted profits.

It will be established in the next subsection that there exists only one solution to these conditions. Section II $C$ will then characterize the 
equilibrium given any state of the system, and Section II $D$ will discuss the stochastic evolution of the economy in light of the properties of $P(K, G)$.

\section{B. Determination of the Equilibrium Price Function}

In this subsection, we will prove that only one price function solves the functional conditions discussed above. Moreover, we will show how this price function can be constructed recursively. Since our goal is to focus on the economics of the model without getting distracted by the mathematics - the inclusion of which is necessary to prove that a unique equilibrium exists and to characterize its properties-we adopt the following approach. We first make five assertions, each of which can be verified by analysis of the functional conditions. Postponing such verification, we treat these assertions as assumptions. We then show why these assumptions imply the existence of a unique solution to the functional conditions. Finally, we verify the validity of the assertions. This last demonstration is graphical and intuitive and occupies most of the subsection.

Consider the following functional conditions:

$$
K-\left\{D\left[P_{n-1}(K, G)\right]+R\left[P_{n-1}(K, G)\right]-G\right\} \geqslant 0
$$

and

$$
\begin{gathered}
P_{n-1}(K, G)-\beta E_{H} P_{n}\left(K-\left\{D\left[P_{n-1}(K, G)\right]\right.\right. \\
\left.\left.+R\left[P_{n-1}(K, G)\right]-G\right\}+\tilde{H}, R\left[P_{n-1}(K, G)\right]\right) \geqslant 0
\end{gathered}
$$

with complementary slackness, where $D(\cdot)$ and $R(\cdot)$, respectively, are the consumer demand curve and government policy correspondence defined previously.

i) Assume that, for each price function $P_{n}(\cdot, \cdot)$ in some restricted class, these conditions can be solved for a unique function $P_{n-1}(\cdot, \cdot)$. Define $T$ as the operator which maps any $P_{n}(\cdot, \cdot)$ in this restricted domain to $P_{n-1}(\cdot, \cdot)$. Then any fixed point of this mapping clearly solves the functional conditions (8)-(9) associated with the infinitehorizon competitive equilibrium. Our strategy will be to show-using contraction-mapping arguments-that only one fixed point exists to this mapping and hence only one solution to the functional conditions. Toward this end, we note the following additional assumptions:

ii) Assume $T$ is an operator defined on the following domain: the set of continuous functions of two variables which are bounded between $\left[0, P^{c}\right]$, nonincreasing in each argument, and no less sensitive to the first than to the second argument. By "no less sensitive" is meant 
that for any function $P(K, G)$ in the domain of $T$, if $K^{\prime}+G^{\prime}=K+G$ and $K^{\prime} \geqslant K$, then $P\left(K^{\prime}, G^{\prime}\right) \leqslant P(K, G)$.

iii) Assume $T$ maps back into its domain.

iv) Assume that if $\hat{P}(K, G)$ and $P(K, G)$ are any two functions in the domain of $T$ and $\hat{P}(K, G) \geqslant P(K, G)$ uniformly, then $T \hat{P}(K, G) \geqslant$ $T P(K, G)$, uniformly.

v) Assume that there exists a $\beta \in[0,1)$ such that for any $\tilde{C}>0$ if $P(K, G)+\tilde{C}$ is in the domain of $T$, then $T[P(K, G)+\tilde{C}] \leqslant T P(K, G)$ $+\beta \tilde{C}$, uniformly.

Then it is easily shown that $T$ is a contraction mapping of modulus $\beta$ with respect to the sup norm. ${ }^{15}$ And since its domain is a closed subspace of a complete metric space and hence is itself a complete metric space (Kelley 1955, p. 192), the classical "principle of contraction mappings" (Kolmogorov and Fomin 1957, p. 43) implies that $T$ has a unique fixed point and that this fixed point can be approximated by beginning with any function in the domain of $T$ and applying $T$ repeatedly. We therefore conclude that the five assumptions suffice to prove the existence of a unique equilibrium price function.

We now verify that each of the five assumptions is in fact an implication of the functional conditions (10)-(11), which define $T$. To begin, we show how $P_{n-1}(\cdot, \cdot)$ can be deduced from any $P_{n}(\cdot, \cdot)$ in the domain of $T$. It is convenient to define the auxiliary function, $X: X=$ $D\left[P_{n-1}(K, G)\right]+R-G$. Substitution of $X$ then permits the following simplification of the conditions defining $T$ :

$$
K-X \geqslant 0
$$

and

$$
D^{-1}(X+G-R)-\beta E_{H} P_{n}(K-X+\tilde{H}, R) \geqslant 0,
$$

with complementary slackness, and

$$
R=R\left(P_{n-1}\right) \text {. }
$$

${ }^{15}$ Blackwell (1965) proved a corresponding proposition for operators defined on a complete linear space. Since our operator is defined only on the closed subset defined in ii, it must be verified that his argument that $T$ contracts remains valid. Denote

$$
\|\hat{P}-P\|=\sup _{K \geqslant 0, G \geqslant 0}|\hat{P}(K, G)-P(K, G)| .
$$

We wish to show that iv and $\mathrm{v}$ imply that $\|T \hat{P}-T P\| \leqslant \beta\|\hat{P}-P\|$ for some $\beta \in[0$, 1). For any pair of functions $\hat{P}(K, G) \leqslant P(K, G)+\|\hat{P}-P\|$, uniformly. If each side of the inequality is in the domain of $T$, we can apply the operator to each side: $T \hat{P}(K, G)$ $\leqslant T[P(K, G)+\|\hat{P}-P\|]$ uniformly by iv. But $T[P(K, G)+\|\hat{P}-P\|] \leqslant T P(K, G)$ $+\beta\|\hat{P}-P\|$, uniformly by v. Hence $T \hat{P}(K, G)-T P(K, G) \leqslant \beta\|\hat{P}-P\|$, uniformly. Reversing the roles of $P$ and $\hat{P}$ and repeating these steps, we obtain $T P(K, G)$ $-T \hat{P}(K, G) \leqslant \beta\|\hat{P}-P\|$, uniformly. This verifies that $\|T \hat{P}-T P\| \leqslant \beta\|\hat{P}-P\|$ for some $\beta \in[0,1)$. Hence $T$ is a contraction mapping of modulus $\beta$ with respect to the sup norm as was asserted in the text. 


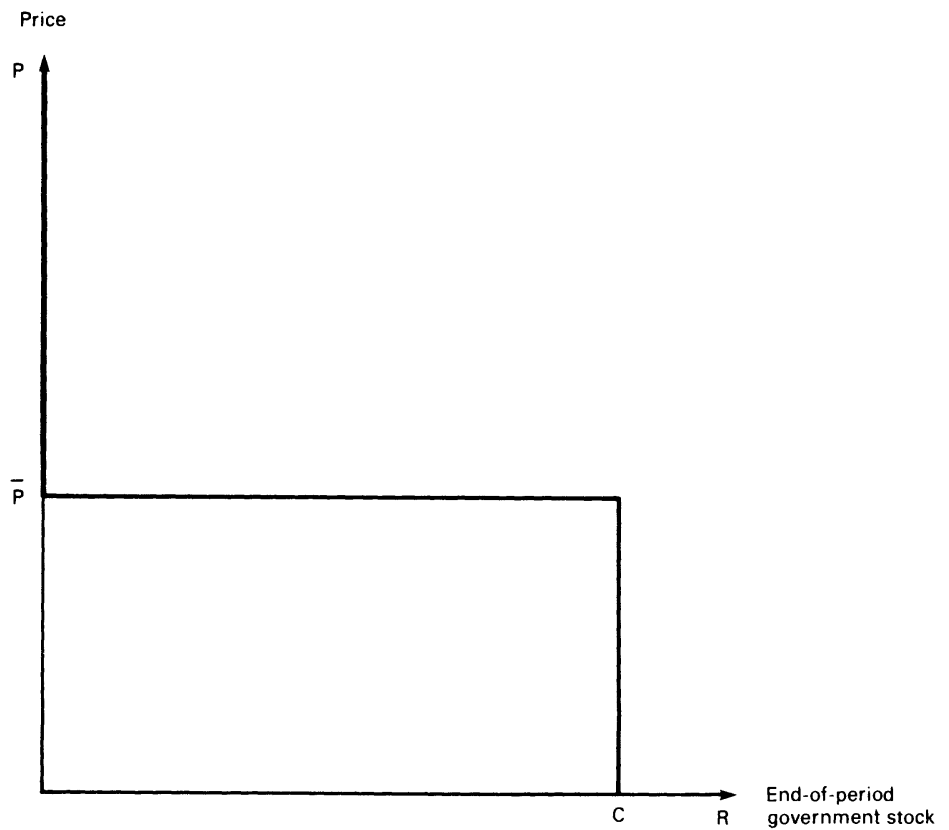

FIG. 3.-The government policy rule of attempted pegging

This last equation defines the relation between $P_{n-1}$ and $R$ which must hold if the government is following its policy rule of price pegging. We portray this nonincreasing relation in figure 3 .

The remainder of this subsection is organized as follows. We will show that the complementary slackness conditions which define $T$ (together with the definition of $X$ ) imply a second relationship between $P_{n-1}$ and $R$-for each $(K, G)$ and admissible starting function $P_{n}(K$, $G)$. This second relationship differs from the first in three important respects. It is derived using $P_{n}(K, G)$. It is sensitive to $(K, G)$. And it is nondecreasing in $R$. This latter characteristic ensures that-for any $(K, G)$-the vertical component $\left(P_{n-1}\right)$ of the point of intersection of the two curves (with $P_{n-1}$ plotted against $R$ ) will be unique. Moreover, as $(K, G)$ is varied the second curve will shift and the unique vertical component $\left(P_{n-1}\right)$ will change. In this way, $P_{n-1}(K, G)$ is traced out.

As noted above, the second relationship between $P_{n-1}$ and $R$ depends on the starting function $P_{n}(K, G)$. If an alternative admissible starting function were used, the derived function $P_{n-1}(K, G)$ would differ. The operator $T$ is simply shorthand to represent the complicated procedure of deriving $P_{n-1}(K, G)$ from $P_{n}(K, G)$. Once this procedure is understood, we conclude the subsection by verifying the validity of what we previously assumed about the operator $T$.

Given $(K, G, R)$ and any $P_{n}(K, G)$ in the domain of $T$, it is 
straightforward to compute $X$ from the complementary slackness conditions. The left-hand side of (13) is the difference of two functions of $X$. The inverse demand curve is-by assumption-continuous, decreasing, and bounded between $\left(0, P^{c}\right]$-with $D^{-1}(0)=P^{c}$. The second function is-by assumption-continuous, bounded between $[0$, $\beta P^{c}$, and nonincreasing in its first argument. Hence the left-hand side of (3) is continuous and decreasing in $X$. To solve the complementary slackness conditions given $(K, G, R)$, provisionally set $X=$ $K$. If the left-hand side of (13) is nonnegative, the conditions are all simultaneously satisfied. If the left-hand side of (13) is negative for $X$ $=K$, lower $X$ until it equals the root of the left-hand side of (13). ${ }^{16}$ This is the alternative way to satisfy the complementary slackness conditions simultaneously. Since the difference of the functions is continuous and decreasing in $X$, there exists a unique solution to (12)-(14) for each $(K, G, R)$. The determination of $X$ is illustrated below in figure 4 .

The variable $X$ has the following economic interpretation. It is the sales (purchases, if negative) which producer-speculators would undertake in aggregate if private and government stocks were initially $(K, G)$, if the government retained $R$ at the end of the period, and if the price function next period were $P_{n}(\cdot, \cdot)$. The left-hand side of (13)-plotted in figure 4-is the discounted loss which each producer-speculator would rationally expect from carrying a unit of stock into the next period given $(K, G, R, X)$ and $P_{n}(\cdot, \cdot)$. The complementary slackness conditions state that producer-speculators will carry no inventories $(K-X=0)$ if they expect a loss $\left(D^{-1}-\beta E P_{n}>\right.$ $0)$; and if they carry positive inventories $(K-X>0)$, no loss is expected $\left(D^{-1}-\beta E P_{n}=0\right)$. These conditions are therefore implications of profit maximization and market clearing. As figure 4 indicates, they imply a unique level of aggregate sales for each $(K, G, R)$ and $P_{n}(\cdot$,$) . An increase in K$ shifts up the expected-loss curve in figure 4. Hence, $d X /\left.d K\right|_{G, R}=1$ if $X=K$, and $X$ increases in $K$ otherwise. An increase in $G$ will shift down the expected-loss curve. Hence $d X /\left.d G\right|_{K, R}$ $\leqslant 0$ if $X=K$, and $X$ decreases in $G$ otherwise. Inspection of the formula for the expected loss reveals one property of particular significance. If $G$ increases by some amount and $K$ decreases by that same amount-so that their sum is unchanged-then $X$ must decrease by that same amount. It can be verified by inspection of (13) that this new $X$ will continue to satisfy the complementary slackness conditions; moreover, since the expected-loss function is decreasing

\footnotetext{
${ }^{16}$ By assumption, the left-hand side of (13) is negative at $X=K$. The existence of a unique root then follows from the fact that the left-hand side of (13) is positive at $X=R$ $-G$ and is continuous and decreasing for all $X$.
} 


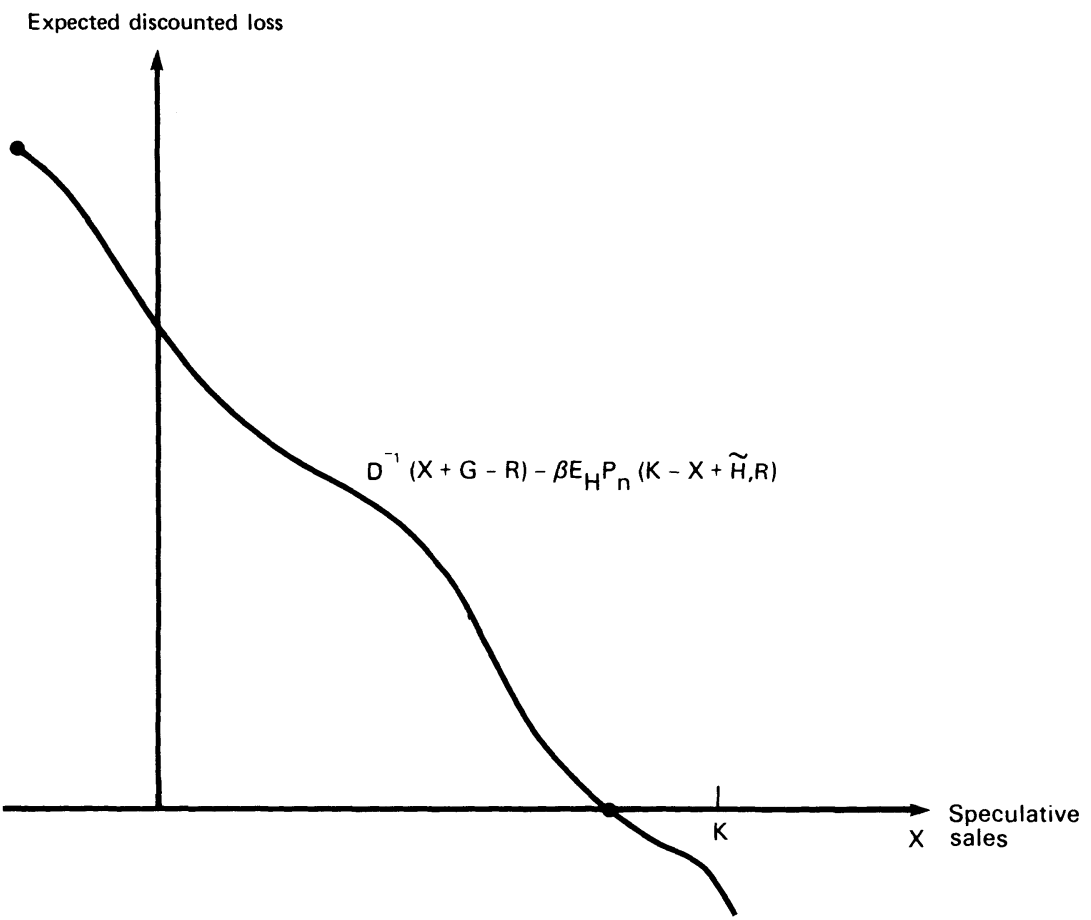

FIG. 4.-Determination of aggregate speculative sales

in $X$, this solution is unique. Hence $d X /\left.d G\right|_{R, K+G}=-1$ for $X \leqslant K$. Finally, since $P_{n}(K, G)$ is assumed to be nonincreasing in its second argument, an increase in $R$ shifts up the expected-loss curve. Hence if $X=K, d X /\left.d R\right|_{K, G}=0$, and $X$ increases in $R$ otherwise. Indeed, our assumption that $P_{n}(\because)$ is "no less sensitive" to its second argument than to its first implies that $0 \leqslant d X /\left.d R\right|_{K, G} \leqslant 1 .{ }^{17}$ This completes the first step outlined above for deriving the nondecreasing relationship between $P_{n-1}$ and $R$.

The second step is less involved. Since $D(\cdot)$ is invertible, we can express $P_{n-1}$ in terms of $(X, G, R): P_{n-1}=D^{-1}(X+G-R)$. By substituting the speculative sales function derived in step one into this inverse demand curve, we can reexpress $P_{n-1}$ as a function of $(K, G$, $R): P_{n-1}=D^{-1}[X(K, G, R)+G-R]$. For the moment, we continue to suppress the dependence of $X(K, G, R)$ and now $P_{n-1}$ on the

${ }^{17}$ In the event that the derivative of $X(K, G, R)$ does not exist somewhere, we can express the corresponding property in terms of first differences. That is, if $R^{\prime}=R+\Delta$ for any $\Delta>0$ and $X^{\prime}=X+\Delta+\epsilon$ then (13) and assumption ii that $P(K, G)$ be "no less sensitive to its first argument than to its second" imply that $\epsilon \leqslant 0$. Hence $\left(X^{\prime}-X\right) /\left(R^{\prime}-\right.$ $R) \leqslant 1$. 
starting function $P_{n}(K, G)$. Since $P_{n-1}$ is a continuous function of a continuous function, it is continuous. Since $D^{-1} \in\left(0, P^{c}\right], P_{n-1} \in(0$, $\left.P^{c}\right]$. Since $d X /\left.d R\right|_{K, G} \leqslant 1, d P_{n-1} /\left.d R\right|_{K, G} \geqslant 0$. For any $(K, G)$, we can therefore plot $P_{n-1}$ as a continuous, nondecreasing function of $R$. In figure 5 below, we plot this second relationship between $P_{n-1}$ and $R$, along with the first relationship from figure 3 .

The vertical component $\left(P_{n-1}\right)$ of their intersection point is unique since one curve is nonincreasing and the other nondecreasing. Thus, $P_{n-1}$ is the unique market price which must result if the government follows its policy rule, producer-speculators maximize expected profits forming their expectations from $P_{n}(\cdot, \cdot)$, markets clear, and stocks at the beginning of the period are $(K, G)$.

As $(K, G)$ is varied, the nondecreasing curve shifts and $P_{n-1}(K, G)$ is traced out; $P_{n-1}(K, G)$ will be continuous and bounded between $\left(0, P^{c}\right.$ ]. Since $X$ increases in $K$, an increase in $K$ (with $G$ held fixed) will shift the nondecreasing curve down for each $R$. Consequently, $P_{n-1}(K, G)$ is nonincreasing in its first argument. If $K$ decreases and $G$ increases by the same amount-so that their sum is constant-then

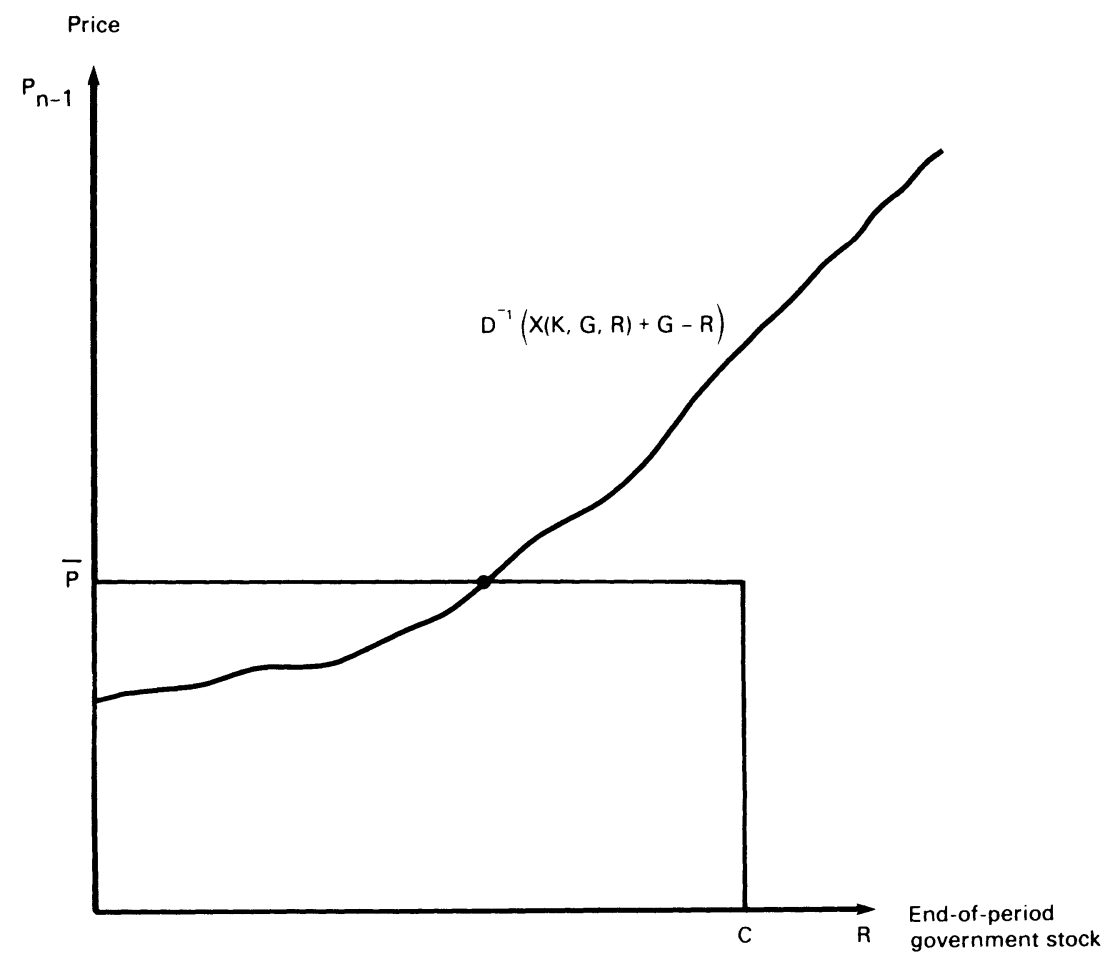

Fig. 5.-Determination of $P_{n-1}(K, G)$ for a particular state of the system 
for fixed $R, X$ will decrease by the same amount as $K\left(d X /\left.d G\right|_{R, K+G}=\right.$ $-1)$. Consequently, for any $R, P_{n-1}$ would be unaffected:

$$
\left.\frac{d P_{n-1}}{d G}\right|_{R, K+G}=D^{-1^{\prime}} \cdot\left(\left.\frac{d X}{d G}\right|_{R, K+G}+1\right)=0 .
$$

Hence $P_{n-1}(K, G)$ is nonincreasing in each argument and equally sensitive to each argument. By "equally sensitive" is meant that if $K^{\prime}$ $+G^{\prime}=K+G$ and $K^{\prime} \geqslant K$, then $P_{n-1}\left(K^{\prime}, G^{\prime}\right)=P_{n-1}(K, G)$.

We conclude this subsection by verifying the validity of each of our five assertions about $T$. We have shown that for any starting function $P_{n}(K, G)$ in the domain of $T$, a unique function $P_{n-1}(K, G)$ is implied by the functional conditions (10)-(11), which define $T$. Furthermore, this image under $T$ will be continuous, nonincreasing in either argument, bounded between $\left(0, P^{c}\right]$, and equally sensitive to its two arguments. Hence $T$ maps back into its domain and assumptions i-iii are verified.

It remains to verify assumptions iv and v. Suppose we had started with a function $\hat{P}_{n}(K, G)$, which was nowhere smaller than $P_{n}(K, G)$. That is, $\hat{P}_{n}(K, G) \geqslant P_{n}(K, G)$ for all $(K, G)$. Then for any $(K, G, R)$ the expected-loss function of figure 4 would have been nowhere higher. Consequently, the speculative sales function derived from the alternative price function $\hat{X}(K, G, R)$ would have been nowhere larger: $\hat{X}(K$, $G, R) \leqslant X(K, G, R)$ and-by substitution- $D^{-1}[\hat{X}(K, G, R)+G-R]$ $\geqslant D^{-1}[X(K, G, R)+G-R]$. Hence, since the nondecreasing relationship between $P_{n-1}$ and $R$ would be nowhere lower, $\hat{P}_{n-1}(K, G) \geqslant$ $P_{n-1}(K, G)$ uniformly. This verifies iv.

Suppose, instead, we had started with the function $\tilde{P}_{n}(K, G)=$ $P_{n}(K, G)+\tilde{C}$, for some $\tilde{C}>0$. Then for any $(K, G, R)$ the expectedloss function of figure 4 would have been shifted down by $\beta \tilde{C}$. Two situations might arise. If, for a particular $(K, G, R), X(K, G, R)<K$, then $\tilde{X}(K, G, R)<K$ and $D^{-1}[\tilde{X}(K, G, R)+G-R]=D^{-1}[X(K, G, R)$ $+G-R]+\beta \tilde{C}$. That is, if, for a given $(K, G, R)$, inventories were carried previously, then an increase of $\beta \tilde{C}$ in the expected discounted price tomorrow would result in an equal increase in the current price. Hence, since the nondecreasing relationship between $P_{n-1}$ and $R$ would be higher by $\beta \tilde{C}, \tilde{P}_{n-1}(K, G) \leqslant P_{n-1}(K, G)+\beta \tilde{C}$. Alternatively, if, for a particular $(K, G, R), X(K, G, R)=K$, then $\tilde{X}(K, G, R) \leqslant K$ and $D^{-1}[X(K, G, R)+G-R] \leqslant D^{-1}[X(K, G, R)+G-R]+\beta \tilde{C}$. That is, if, for a given $(K, G, R)$, inventories were not previously carried, an increase of $\beta \tilde{C}$ in the expected discounted price would raise the current price by no more than $\beta \tilde{C}$. In this case too, $\tilde{P}_{n-1}(K, G) \leqslant P_{n-1}(K$, $G)+\beta \tilde{C}$. This verifies v.

We conclude, therefore, that a unique price function solves the equilibrium conditions of our model. Moreover, a similar approach 
can be used to show that a unique price function exists for the case of a price band. ${ }^{18}$ In the next subsection, we study the properties of the equilibrium under price pegging for any state of the system.

\section{Properties of the Equilibrium with Attempted Pegging for Any State of the System}

In this subsection, we study the key aspects of the equilibrium associated with each pair of stocks $(K, G)$. We first note that the market price and hence end-of-period government stock can be expressed as a function of the sum of private and government stocks $(K+G)$. We study properties of each of these functions and discover, once again, an attack threshold. If the combined stock falls beneath this threshold, speculators purchase the entire government stock and the market price breaks through the ceiling. In the final subsection we will study the stochastic evolution of the economy and the odds that such attacks occur and recur.

Recall that the existence of a unique equilibrium price function was established in Section IIIB. This price function is the fixed point of the mapping $T$. Since any image of $T$ is equally sensitive to its two arguments and the equilibrium price function is the image (of itself) under $T, P(K, G)$ must possess this property of equal sensitivity. Hence, we can express the equilibrium price as a nonincreasing function of a single variable - the sum of the private and government stocks. Denote this sum by $\theta$. That is, $\theta=K+G$. Since the fixed point is equally sensitive to its two arguments, the speculative sales function derived from it $(X[K, G, R])$ has the property that $\left.(d / d R) X(K, G, R)\right|_{K, G}$ $=1$ for $X(K, G, R)<K$. That is, for any $(K, G)$, increases in $R$ result in equal increases in $X$ until $X$ hits its maximum value $(K)$ and can increase no further. This property of the speculative sales function in

${ }^{18}$ If the government attempted to defend a real price band, we could express its policy correspondence as follows:

$$
\begin{aligned}
& \begin{array}{lll}
R=0 & \text { if } \quad P>P_{u}
\end{array} \\
& \begin{array}{lr}
R \in[0, G] & P=P_{u} \\
R=G & P_{l}<P<P
\end{array} \\
& R \in[G, C] \quad P=P_{l} \\
& R=C \quad P<P_{l},
\end{aligned}
$$

where $P_{u}$ and $P_{l}$ denote the upper and lower limits of the band $\left(P_{u} \geqslant P_{l}\right)$. If the limits are equal, the policy is identical to attempted pegging; if $P_{u}=P^{c}$ and $P_{l}=0$, the policy is laissez-faire. It should be noted that this new government policy can be represented by a different, nonincreasing correspondence in $P-R$ space (for a given $G$ ) and that profitmaximizing speculative behavior would give rise to the same nondecreasing curve for each $(K, G)$ as with a peg (given the same price function next period). However, in the case of a band, the property of equal sensitivity is not inherited by successors in the sequence of price functions. Hence the infinite-horizon equilibrium price function cannot be expressed as a function of the sum of private and public stocks. 
turn affects the shape of the nondecreasing function in figure 5 . For any $(K, G)$ as $R$ increases from zero, $X$ increases at the same rate as $R$; hence $D^{-1}[X(K, G, R)+G-R]$ is unaffected. That is, $\left.(d / d R) D^{-1}[X(K, G, R)+G-R]\right|_{K, G}=0$, for $X(K, G, R)<K$. Once $X$ $=K, d D^{-1}(\cdot) /\left.d R\right|_{K, G}=D^{-1^{\prime}}(K+G-R)>0$. Thus, for any $(K, G)$, as $R$ increases, the function remains constant for some interval, kinks, and then increases thereafter. Along the increasing segment, $X=K$ and $P=D^{-1}(\theta-R)$. The increasing segment is therefore simply a laterally displaced mirror image of the inverse demand curve (for $P>$ $\bar{P})$. When $R=\theta$, the increasing segment equals $D^{-1}(0)=P^{c}$. Thus, whenever the equilibrium occurs on the horizontal portion of the kinked curve, $X<K$ and speculators carry inventories; whenever it occurs on the increasing segment of the kinked curve, $X=K$ and speculators carry zero inventories. The size of the combined stock $(K$ $+G=\theta$ ) underlying any particular kinked curve can be determined by noting where it intersects the horizontal line of height $P^{c}$.

Figure 6 depicts the family of these kinked curves and is derived from the equilibrium price function. Higher values of $\theta$ are associated with lower curves. I have drawn the increasing segment of each kinked curve as linear for greater legibility but make no use of this characteristic. ${ }^{19}$ Some price functions in figure 6 emanate from the vertical axis with a horizontal slope and then are kinked, while others emanate with a positive slope and have no kinks. It can be shown in general that the locus of kinks rises to the left until it intersects the vertical axis, and that the price functions with higher vertical intercepts have no kinks. ${ }^{20}$

Figure 6 also contains the nonincreasing government policy correspondence of figure 3 . As $\theta$ increases from zero, the kinked curvewhich depends on $\theta$-shifts down and its point of intersection with the government policy rule changes.

I record the derived information in the two panels of figure 7 . In the first panel, I plot against $\theta$ the vertical component of the points of intersection, $P(\theta)$. In the second panel, I plot against $\theta$ the horizontal component of the points of intersection, $R[P(\theta)]$. These two panels record the market price and end-of-period government stock associated in equilibrium with each $\theta$. As can be seen in figure 6 , if $\theta=$ $0, P(\theta)=P^{c}$ and $R[P(\theta)]=0$. As $\theta$ increases, $P(\theta)$ declines and

\footnotetext{
${ }^{19}$ In fact, the increasing segment will be linear if and only if the inverse demand curve is linear for $P \geqslant \bar{P}$.

${ }^{20}$ Each price function is kinked where $X=K$ and $D^{-1}(X+G-R)=\beta E P(K-X+$ $H+R)$ or $D^{-1}(\theta-R)=\beta E P(H+R)$. Since $d R / d \theta>0$, the locus of kinks $(N M)$ in fig. 6 is downward sloping. To show that price functions above the point where the locus of kinks cuts the vertical axis emanate with a positive slope, note that if $R=0$ and $G$ is reduced (for a fixed $K$ ), $X$ must remain at $K$ (since $d X /\left.d G\right|_{K, R}=0$, for $X=K$ ).
} 


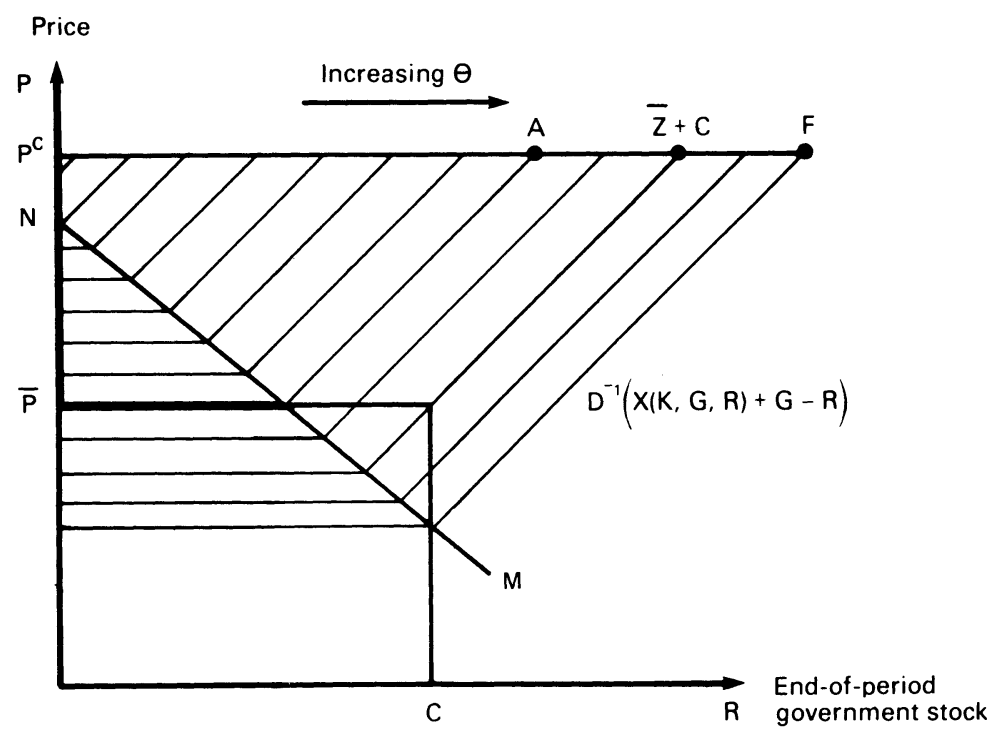

Fig. 6.-Determination of the market price and end-of-period government stocks for any state of the system.

$R[P(\theta)]$ remains zero. Eventually, $\theta$ reaches a threshold, denoted $A$, such that $P(\theta)=\bar{P}$ for $\theta=A$ and $P(\theta)>\bar{P}$ for $\theta<A$. At this point $R[P(\theta)]$ is indeterminate. The indeterminacy arises because the horizontal segment of the kinked curve coincides with the horizontal segment of the government policy rule. Denote the demand induced by the official price as $\bar{Z}$. That is, $D(\bar{P})=\bar{Z}$. Then $P=D^{-1}(X+G-R)$ $=\bar{P}$ or $R=X+G-\bar{Z}$. In the indeterminacy, $X$ varies between $Z-$ $G$ and $K$; correspondingly, $R$ varies between 0 and $A-Z$. As $\theta$ increases beyond $A, P(\theta)=\bar{P}$. Since the increasing segment of the kinked curve intersects the policy rule, $X=K$ in this range and no private stocks are carried. Since $D^{-1}(K+G-R)=\bar{P}, R=\theta-\bar{Z}$. Hence as $\theta$ increases, $R$ increases at the same rate and $P(\theta)=\bar{P}$. Eventually, $R=C$ and can increase no further. At that point $\theta=\bar{Z}+$ $C$. For $\theta>\bar{Z}+C, P(\theta)=D^{-1}(\theta-C)<\bar{P}$ and $R[P(\theta)]=C$. This information is depicted in the two panels of figure 7 .

Figures 6 and 7 are not completely general. The appearance of the curves in figure 7 would be different if the locus of kinks $(N M)$ in figure 6 did not cut $\bar{P}$ at some $R \in(0, C)$. If the locus cut $\bar{P}$ for $R \geqslant C$, $A=Z+C$. Consequently the horizontal segment of the price function in figure $7 a$ and the positively sloped portion of the curve in $7 b$ would disappear. Note the minor differences which arise in this case in footnotes 22 and 23. It is also possible that the locus of kinks does not cut $\bar{P}$ for any $R \geqslant 0$. This cannot occur in a continuous-time 

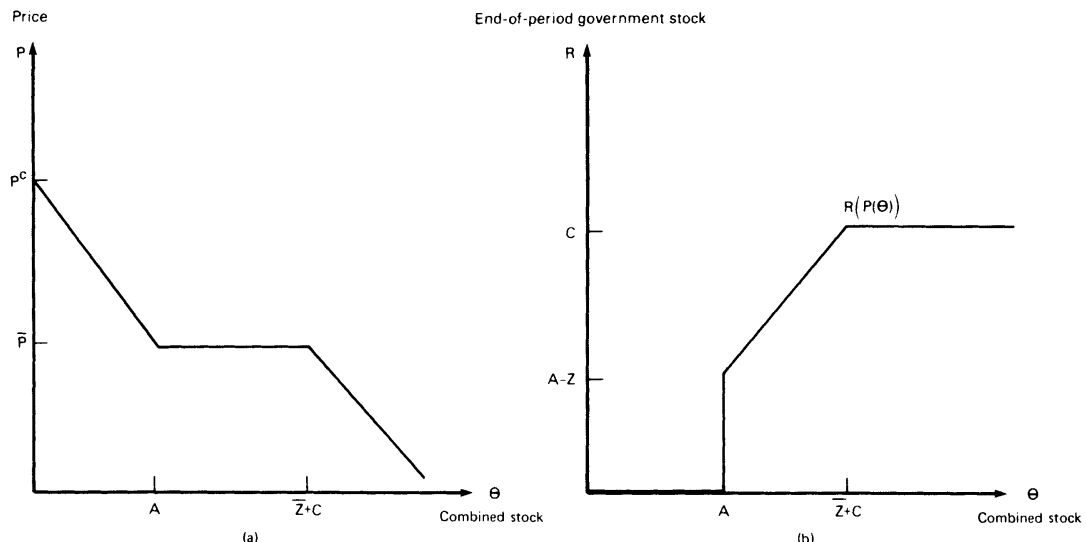

FIG. 7.-The equilibrium price and end-of-period government stock resulting from alternative combined stocks at the beginning of a period.

treatment and arises in discrete analysis only if the official price is set very close to the choke price. A similar case arose in Section II. As we did there, let us impose a weak assumption on the relationship of the official price to the choke price that ensures that this case cannot occur. $^{21}$

The two curves in figure 7 describe many aspects of the equilibrium. As long as the combined stocks are neither too large nor too

${ }^{21}$ If the locus of kinks does not lie above $\bar{P}$ for $R \geqslant 0$, then $D^{-1}(X+G-R) \leqslant \bar{P}$ and $D^{-1}(X+G-R)-\beta E P(K-X+\tilde{H}+R) \geqslant 0$ for $R=0$ and $X=K$. Hence, $\bar{P} \geqslant$ $D^{-1}(\theta) \geqslant \beta E P(\tilde{H})$. That is, the case we wish to rule out occurs only if the official price is set so high that-even if no private or government inventories were carried into the next period-there would still exist no incentive for a speculator to pay so high a price to acquire one unit of stocks. To rule out this uninteresting case, we must restrict the height of $\bar{P}$ so that $\bar{P}<\beta E P(\tilde{H})$. A stronger condition can be stated in terms of the givens of the problem. Define $P_{N}(K, G)=T \cdot 0$. Since it is the image of the operator $T$, it will be equally sensitive to its two arguments. Hence we can express the market price as a function of the sum, $K+G=\theta$ :

$$
P_{N}(\theta)= \begin{cases}D^{-1}(\theta) & \text { for } 0 \leqslant \theta<\bar{Z} \\ \bar{P} & \bar{Z}+C \geqslant \theta \geqslant \bar{Z} \\ D^{-1}(\theta-C) & \theta>\bar{Z}+C\end{cases}
$$

Hence

$$
\beta E P_{N}(\tilde{H})=\beta\left\{\int_{0}^{\bar{Z}} D^{-1}(H) d F(H)+\bar{P} \int_{\bar{Z}}^{\bar{z}+C} d F(H)+\int_{\bar{Z}+C}^{\infty} D^{-1}(H-C) d F(H)\right\} .
$$

The stronger restriction referred to above is that $\beta E P_{N}(\tilde{H})>\bar{P}$. We will now show that if $\beta E P_{N}(\tilde{H})>\bar{P}$, then $\beta E P(\tilde{H})>\bar{P}$. To prove that $\bar{P}<\beta E P_{N}(\tilde{H})$ implies that $\bar{P}<$ $\beta E P(\tilde{H})$, we first show that $P(\theta) \geqslant P_{N}(\theta)$, where $P_{N}(\theta)=T 0(\theta)$. Since $P_{N}(\theta)>0$ and $T(\cdot)$ satisfies iv, $T^{2} 0(\theta) \geqslant T 0(\theta)$ or $T^{n+1} 0(\theta) \geqslant T^{n} 0(\theta) \geqslant T 0(\theta)$. Hence, $\lim _{n \rightarrow \infty} T^{n} 0(\theta) \equiv P(\theta)$ $\geqslant T 0(\theta) \equiv P_{N}(\theta)$. But if $P(\theta) \geqslant P_{N}(\theta), \beta E P(\tilde{H}) \geqslant \beta E P_{N}(\tilde{H})$ and the claim is established. In Sec. II, the harvest was always zero and the restriction that $\beta E P(H)>P$ reduces to $\beta P^{c}>\bar{P}$. This condition is discussed in n. 10 . 
small $(A<\theta<\bar{Z}+C)$, the government can peg the market price. But if $\theta>Z+C$, the government stockpile cannot be enlarged and the official price cannot be supported. Hence $P<\bar{P}$. Alternatively, if $\theta<$ $A$, the government cannot defend the ceiling $(P>\bar{P})$ despite its liquidation of its entire stock of reserves. Note that as $\theta$ crosses $A$ from above, the end-of-period government stock drops precipitously. Since consumption is a continuous function $(D[P(\theta)])$ of $\theta$, this sudden reduction in government stocks has as its counterpart a sudden acquisition by producer-speculators. Hence, a speculative attack occurs when $\theta$ drops below $A$. For this reason, we refer to $A$ as the attack threshold. ${ }^{22}$

Figure 6 depicts one other phenomenon of interest. As $\theta$ is increased beyond $\bar{Z}+C$, the market price falls beneath $\bar{P}$. At first the intersections of the relevant kinked curves and the policy rule occur in the increasing segment of the kinked curves. ${ }^{23}$ Beyond some stock, denoted $F$ in figure 6 , the intersections occur on the horizontal segment of the kinked curves. ${ }^{24}$ Hence, for $F>\theta>\bar{Z}+C$ speculators carry no stocks between periods. For $\theta>F$, however, they carry stocks. The behavior of speculators when $F>\theta>\bar{Z}+C$ is easily understood. For $\theta$ in this region, the market price is only slightly below the official ceiling price. Thus, the government policy truncates capital gains but not capital losses. Under this circumstance, it is not profitable for speculators to carry stocks. If social stocks exceed $F$, however, the market price is depressed enough below the ceiling that the potential capital gains attract speculation. Similar properties are to be expected in models with price bands. Speculators will hold no stocks when the market price is slightly below a ceiling but will hold inventories when the market price is slightly above a floor. Such situations are often labeled "one-sided bets" against breaches of the ceiling.

Given $P(\theta)$, we can compute the discounted expected price in the following period as a function of $\theta: \beta E P\{\theta+\tilde{H}-D[P(\theta)]\}$. Current and discounted expected price are plotted together in figure 8 . For any $\theta$, the discounted expected price either equals or is less than the

${ }^{22}$ If the locus of kinks $(N M)$ equals $\bar{P}$ for $R>C$, the government is never able to defend its ceiling and an attack occurs the moment $\theta$ falls below $\bar{Z}+C$.

${ }^{23}$ In the case where the locus of kinks $(N M)$ equals $\bar{P}$ for some $R>C$, speculators would willingly hold stocks for any $\theta>\bar{Z}+C$. In this case, the equilibrium price function has no horizontal segment, reflecting the complete inability of the government to defend the ceiling. Speculators are willing to carry stocks even when the market price is just under $\bar{P}$ because they know the government will be unable to defend the ceiling and truncate their capital losses.

${ }^{24}$ Implicitly, $F$ may be defined as that $\theta$ which solves the equation $D^{-1}(\theta-C)=$ $\beta E P(\tilde{H}+C)$. 
Expected discounted price, current price

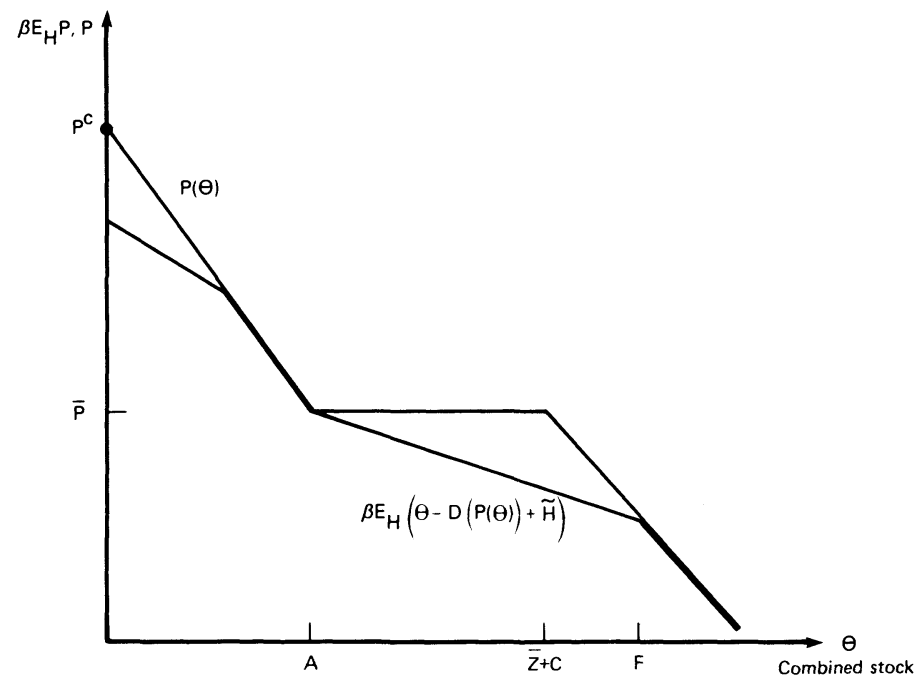

FIG. 8. - The current and expected discounted price next period for any state of the system.

current price. Whenever a capital loss is expected (e.g., when $A<\theta<$ $F)$, speculators carry zero inventories $(K-X=0)$.

Figure 8 can be used to clarify an important question. One mystery of speculative attacks is why there is never a range of social stocks for which the government maintains the official price while speculators hold stocks. Why is there only one size of social stock which induces this behavior?

If, for some range of $\theta$, speculators held stocks while the government maintained the official price, then the discounted expected price would have to equal the official price over that range of $\theta$. But as figure 9 illustrates ${ }^{25}$ the expected discounted price function is strictly decreasing when its height is $\bar{P}$ and is nonincreasing throughout. Hence only one size of social stock is consistent with private holding at the official price. A similar result holds for any government policy with a ceiling.

${ }^{25}$ The argument is made for $H$ a discrete random variable but can be extended for cases where $H$ is continuous. The term $\beta E P\{\theta+H-D[P(\theta)]\}$ can be regarded as the discounted, weighted sum of functions of $\theta$. Each function is similar in shape to fig. $7 a$ but is horizontally displaced by $H-[D P(\theta)]$. Consequently, each function strictly decreases when its value differs from $\bar{P}$ and is constant (or nondifferentiable) when its value equals $\bar{P}$. By assumption, the mean discounted price next period equals $\bar{P}$. Accordingly, at least one function in the discounted weighted average must exceed $\bar{P}$ and the discounted mean price must therefore be strictly decreasing in $\theta$ when its value equals $\bar{P}$. Reversing the argument, the discounted expected price function can have a zero derivative with respect to $\theta$ only when the function has height $\beta \bar{P}$. 


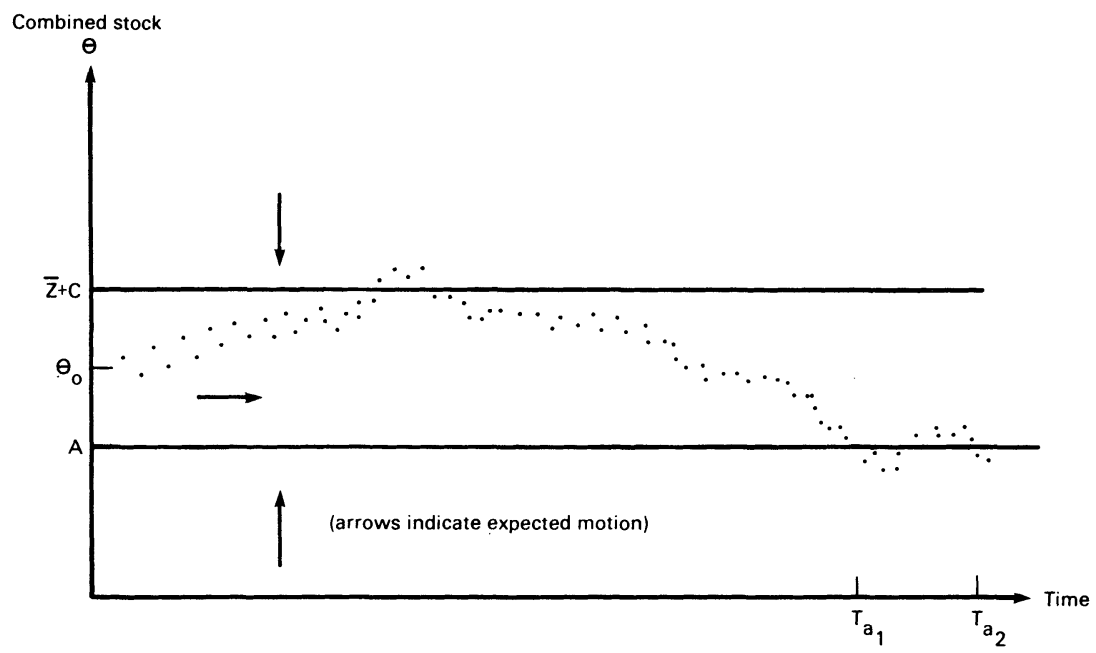

FIG. 9.-One realization with two speculative attacks indicated

\section{Stochastic Evolution of $\theta$}

It has been shown that a unique limiting price function exists and that it exceeds or falls short of the official price depending on the magnitude of $\theta$. Three regions may be distinguished:

$$
\begin{array}{lll}
\text { region } 1 & \theta<A & {[P(\theta)>\bar{P}] ;} \\
\text { region 2 } & \bar{Z}+C \geqslant \theta \geqslant A & {[P(\theta)=\bar{P}] ;} \\
\text { region 3 } & \theta>\bar{Z}+C & {[P(\theta)<\bar{P}] .}
\end{array}
$$

Transitions from one region to its neighbor have the following economic interpretation. If $\theta$ crosses from region 2 to region 1 , the government loses its entire stockpile in a sudden attack and can no longer prevent the market price from exceeding the official level. As long as $\theta$ remains in region 1 , all stocks carried between periods are held by private speculators. When $\theta$ crosses back into region 2 , speculators expect inadequate gains and sell their stocks to the government at the official price. As long as $\theta$ remains in region 2, all stocks carried between periods are held by the government. When $\theta$ crosses from region 2 to region 3 , the government is no longer able to purchase the additional grain necessary to support the market price and it falls below the official level. As long as $\theta$ remains in region 3, the government continues to carry its full capacity. If additional stocks are carried they must be held by private speculators. When $\theta$ crosses back into region 2, all stocks are once again carried by the government. 
To complete the analysis, let us study the stochastic evolution of $\theta$ according to the following first-order Markov process:

$$
\begin{gathered}
P_{t}=P\left(\theta_{t}\right), \\
\tilde{\theta}_{t+1}=\theta_{t}+\left[\tilde{H}_{t+1}-D\left(P_{t}\right)\right], t=1,2, \ldots, \\
\theta_{1}=\tilde{\theta} .
\end{gathered}
$$

Price Function

Transition Equation

Initial Condition

Various events of economic interest depend on $\theta$ and it is sometimes possible (even without Monte Carlo simulation) to compute the probabilities that events of particular interest occur. To illustrate, we will investigate the probability that $\theta$ eventually declines below $A$ regardless of the initial size of the private plus government stocks and also the probability that this event will recur "frequently." We will assume that the official price is set to induce consumption equal to the expected harvest $(D[\bar{P}]=E[\tilde{H}])$. Consider the stochastic difference equation above. If it were true that $P(\theta)=\bar{P}$ for all $\theta, \theta$ would execute an ordinary, additive random walk with zero drift. In fact, although $P(\theta)=\bar{P}$ for $\theta \in[A, \bar{Z}+C]$, the function is decreasing elsewhere. When stocks exceed $\bar{Z}+C$, the price falls-stimulating additional consumption; when stocks fall below $A$, the price rises-rationing consumption. Hence the stochastic process under consideration is a variant of the much-studied classical random walk ${ }^{26}$ and can be analyzed by using well-known results.

A typical realization of $\theta_{t}$ is represented graphically in figure 9 . The question of whether an attack must occur is equivalent to the question of whether $\theta$ must eventually drop below the attack threshold. The question of its recurrence is equivalent to whether with "virtual certainty" $\theta$ drops below the attack threshold more than any finite number of times.

The claim that a speculative attack is virtually inevitable rests on the standard theorem in random walk theory popularized in the proposition that playing a fair game against an opponent with infinite wealth "assures" ruin. That is, in $n$ rounds of play, various distinct ordered sequences of events (wins and losses) might occur. Each sequence of length $n$ has a probability attached to it. The sum of the probabilities of events (sequences) which imply ruin (zero wealth) approaches one for large $n$.

Little need be changed to apply this theorem to our modified random walk. Assume for the moment that the disturbance term $\left(\tilde{H}_{t}-\right.$ $\left.D\left[P\left(\theta_{t}\right)\right]\right)$ conformed exactly to the conventional random walk as-

\footnotetext{
${ }^{26} \mathrm{By}$ a classical random walk is meant any process of the form $X_{t+1}=X_{t}+\tilde{\mu}$, where $\tilde{\mu}$ is i.i.d.; $\bar{\mu}$ can be either a discrete or a continuous random variable. The extension of the usual theorems for discrete states to the case of continuous states is due to the work of Chung and Fuchs (1951).
} 
sumption (zero mean, i.i.d.). Then $\theta$ would hit zero with probability one-and would, a fortiori, also hit the attack threshold. More precisely, for any $n$, some sequences of $n$ harvests would cause the stock to drop below the attack threshold and some would not, but the probability of generating sequences in the former class can be made arbitrarily close to one by suitable choice of $n$. For any $n$, consider the set of sequences of harvests which would cause $\theta$ to cross the attack threshold. All such sequences would still generate attacks if proper account is now taken of the fact that-when combined stocks exceed $\bar{Z}+C$-the additive disturbance term should in fact be smaller than was supposed when the additional induced demand was neglected. Indeed, some sequences of harvests which did not result in a crossing of the threshold when the additional component of demand was ignored would result in an attack when proper account of it is taken. Hence, for any $n$, the probability of an attack is at least as great in the case of our modified random walk as in the conventional case. This proves that if the official price induces demand equal to (or exceeding) the expected harvest, the buffer stock manager will almost surely experience a speculative attack. As for the possibility of repeated attacks, Wenocur and Salant (1981) extend the recurrence property of the discrete-time, continuous-state, classical random walk with zero drift to the case of our modified random walk. They demonstrate that if the demand induced by the official price exactly matches the expected harvest, the buffer stock manager can look forward with virtual certainty to an infinite number of subsequent attacks.

\section{Conclusion}

In this paper, we have considered situations where the government is unable to use a buffer stock to stabilize a price forever. Its futile attempts to do so result either in upward price jumps if speculators are absent or in speculative attacks if speculators are present. The economic function of such attacks deserves emphasis. An attack prevents the occurrence of dislocations which result from sudden cutbacks in consumption. Such cutbacks accompany upward jumps in price.

One general consequence of competitive speculation is the elimination of all upward price jumps which can be foreseen. ${ }^{27}$ When the government stocks cross a threshold beyond which a future price jump would be expected, the prospective jump is avoided by the swift "transfer" of stocks of the commodity that has become scarce from the hands of the buffer stock manager, who would continue to sell the

${ }^{27}$ In this sense at least, speculators $d o$ stabilize prices. 
remaining reserves at the low official price, to speculators who will sell them at market prices which reflect economic scarcity. The attack is instantaneous because of the linearity of the government sales rule and the fact that speculators in aggregate can make rapid acquisitions at a constant per unit cost.

Whether such attacks actually occur under the circumstances specified in this paper is an empirical matter which no amount of theorizing can resolve. The predictions of the paper follow logically from the assumptions. Readers who doubt the validity of the assumptions concerning optimal behavior or rational expectations may question the validity of the theoretical predictions. Nonetheless, it should be emphasized that although these assumptions have been shown to be sufficient to generate the conclusions, there has been no contention that they are necessary. On the contrary, similar conclusions may well follow from more "realistic" assumptions.

Like any other, this theory should be judged by the accuracy of its predictions. Fortunately, experiments have been designed and executed (Miller, Plott, and Smith 1977) that strongly support the predictions of the theory of intertemporal competitive speculation in the absence of government intervention. To extend these laboratory experiments to situations involving attempted pegging seems straightforward. Such an extension would permit a replicable test of the theory.

If the theory predicts well, a computerized version of the modelgeneralized to permit analysis of ceilings, floors, and bands-could be used to forecast the consequences of each of the many proposed buffer stock schemes.

\section{Appendix A}

\section{On General Equilibrium Interpretations of the Hotelling Model}

The purpose of this Appendix is to reassure readers unfamiliar with the Hotelling model that there do exist at least two general equilibrium models which give rise to the special interest rate and demand curve assumptions of the Hotelling model of Sections II and III.

Since the first model is described at length in the literature on exhaustible resources (Dasgupta and Heal 1979), it is mentioned only briefly. The second model, alluded to in several articles by Samuelson among others, is described in more detail.

In model 1, a single output is produced using the resource as an input. Production requires the contemporaneous depletion of the input. Both the resource and the produced output are storable. One unit of stored output is assumed to result in $1 / \beta$ units of output in the next period, reflecting a constant marginal rate of transformation through roundabout production. Consumers rank time-dated bundles of consumption of the output using a 
quasi-concave utility indicator and select the most preferred bundle which they can obtain at given prices. To acquire this bundle, they must maximize the market value of sales from their resource endowment and stored output.

In this model, as long as output is stored, one unit of output today must exchange on the market for $1 / \beta$ units of output tomorrow. Hence the output rate of interest is $(1 / \beta)-1$, a constant. ${ }^{28}$ The "demand curve for the resource" is a derived demand for an input (that amount of input the marginal product of which equals the real factor price) and therefore depends on the price of the input relative to the contemporaneous price of the output. Hence the factor demand curve is independent of other prices, wealth, production in other periods, and so forth.

In model 2, there is no production. The economy consists of consumers with initial stocks of two consumable, storable goods- $Z$ and $Y$-and subsequent endowments of $Y$ in each period. For simplicity, assume all consumers are identical. Their utility function has the following form:

$$
U\left(z_{1}, z_{2}, \ldots, z_{T} ; y_{1}, y_{2}, \ldots, y_{T}\right)=\sum_{t=1}^{T}\left[f\left(z_{t}\right)+a y_{t}\right] \beta^{t-1},
$$

where $z_{t}$ and $y_{t}$ are the consumption in period $t$ of the two goods. We refer to $Y$ as the "background good"; it is notable because consuming additional amounts of it in a period yields constant increases in utility. The marginal rate of substitution between $Y$ in adjacent periods is therefore constant $(1 / \beta)$.

The consumer takes as given the price sequences for $Z$ and for $Y:\left\{P_{t}^{z}\right\},\left\{P_{t}^{y}\right\}$. By transforming his endowments through storage and exchanges with the market, the consumer acquires his most preferred consumption bundle. His feasible set of consumption opportunities is defined as follows:

$$
\sum_{t=1}^{T} P_{t}^{z} z_{t}+\sum_{t=1}^{T} P_{t}^{y} y_{t} \leqslant W
$$

where

$$
\begin{gathered}
W=\sum_{t=1}^{T} P_{t}^{z} x_{t}^{z}+\sum_{t=1}^{T} P_{t}^{y} x_{t}^{y}, \\
K_{1}^{z}=\bar{K}^{z}, K_{1}^{y}=\bar{K}^{y}, \\
K_{t+1}^{z}=K_{t}^{z}-x_{t}^{z} ; K_{t+1}^{y}=K_{t}^{y}-x_{t}^{y}+\bar{e}_{t}^{y},
\end{gathered}
$$

and

$$
K_{t}^{z} \geqslant 0, K_{t}^{y} \geqslant 0, z_{t} \geqslant 0, y_{t} \geqslant 0 ;
$$

$P_{t}^{z}$ denotes the price per unit of $Z$ in period $t, P_{t}^{y}$ denotes the price per unit of $Y$ in period $t, x_{t}^{z}$ denotes the sales (purchases if negative) of $Z$ in period $t, x_{t}^{y}$ denotes the sales (purchases if negative) of $Y$ in period $t, \bar{e}_{t}^{y}$ denotes the endowment of $Y$ in period $t, K_{t}^{z}$ denotes the stock of $Z$ remaining in period $t$ prior to sales then, $K_{t}^{y}$ denotes the stock of $Y$ remaining in period $t$ prior to sales then, and $W$ denotes the value of market sales.

By a well-known separation theorem, the maximization of consumer utility

${ }^{28}$ Since there is another good in model 1 , there is a second interest rate. The input rate of interest is zero as long as the resource is stored and nonpositive in any case. 
can be accomplished in two steps. First, $W$ is maximized subject to (A.1)-(A.4) by choosing $x_{t}^{z}, x_{t}^{y}, K_{t}^{z}$, and $K_{t}^{y}$ optimally. Then the best consumption bundle is chosen, given the maximized $W$. We begin by considering this latter maximization problem.

If $\left(z_{1}, z_{2}, \ldots, z_{T} ; y_{1}, y_{2}, \ldots, y_{T}\right)$ is the most preferred consumption bundle, then it must satisfy the following Kuhn-Tucker conditions for some $\lambda>0$ :

$$
z_{t} \geqslant 0, \beta^{t-1} f^{\prime}\left(z_{t}\right)-\lambda P_{t}^{z} \leqslant 0,
$$

with complementary slackness, and

$$
y_{t} \geqslant 0, \beta^{t-1} a-\lambda P_{t}^{y} \leqslant 0
$$

with complementary slackness. "Complementary slackness" means that at least one of the two inequalities must hold with equality. Consider any arbitrary sequence of positive prices of $Y,\left\{P_{t}^{y}\right\}$. From (A.6), positive amounts of $Y$ will be consumed only in those periods where $P_{t}^{y} / a \beta^{t-1}$ is smallest. In other periods, $y_{t}=0$.

Next, consider the problem of choosing $x_{t}^{z}, x_{t}^{y}, K_{t}^{z}$, and $K_{t}^{y}$ to maximize wealth. If $P_{t+1}^{z}-P_{t}^{z}>0$ or $P_{t+1}^{y}-P_{t}^{y}>0$ for any $t$, the wealth maximization problem has no solution. For it would then always be possible to increase wealth further by buying more in period $t$, storing the extra amount, and selling it in period $t+1$. Indeed, attempts to do so on a grand scale are what ensure that neither inequality would hold in equilibrium.

If neither inequality holds, the wealth maximization problem does have a solution. If $\left(K_{1}^{z}, K_{2}^{z}, \ldots, K_{T+1}^{z} ; K_{1}^{y}, K_{2}^{y}, \ldots, K_{T+1}^{y}\right)$ is chosen to maximize wealth, then the following Kuhn-Tucker conditions must hold for $t=1,2, \ldots, T$ :

$$
K_{t+1}^{z} \geqslant 0, P_{t+1}^{z}-P_{t}^{z} \geqslant 0
$$

with complementary slackness;

$$
K_{t+1}^{y} \geqslant 0, P_{t+1}^{y}-P_{t}^{y} \geqslant 0,
$$

with complementary slackness.

Together, these conditions imply that, in any equilibrium, $Y$ is never stored and $P_{t}^{y}=\beta^{t-1} P_{1}^{y}$. For suppose that $Y$ were stored at $t$. It is clearly not optimal to store $Y$ beyond the end of the horizon. Suppose instead storage terminates earlier. Specifically, let $t+m$ be the final period of storage. By (A.8), $P_{t}^{y}=$ $P_{t+1}^{y}=P_{t+2}^{y}=\ldots=P_{t+m}^{y}$, for some integer $m \in[1, T-t]$. But then $\left(P_{t}^{y} /\right.$ $\left.a \beta^{t-1}\right)<\left(P_{t+1}^{y} / a \beta^{t}\right)<\left(P_{t+2}^{y} / a \beta^{t+1}\right)<\ldots<\left(P_{t+m}^{y} / a \beta^{t+m-1}\right)$, and, by (A.6), $y_{t+1}=y_{t+2}=\ldots=y_{t+m}=0$. Hence there would be zero demand for $Y$ in periods $t+1, \ldots, t+m$. But since the stored $Y$ is carried into these periods and, by assumption, is not carried beyond $t+m, Y$ will be offered for sale during this time interval, creating excess supply of $Y$. Thus, storage of $Y$ inevitably results in a disequilibrium.

Hence, in equilibrium, $Y$ will not be stored. Since, however, there are endowments of $Y$ in every period, prices must adjust to induce positive consumption of $Y$ in every period.'Equation (A.6) implies that if $y_{t}>0$ in every period, $P_{t}^{y}=\beta^{t-1} P_{1}^{y}$. Since $P_{t}^{y} / P_{t+1}^{y}$ is the amount of $Y$ obtainable at $t+1$ in exchange for one unit of $Y$ at $t,\left(P_{t}^{y} / P_{t+1}^{y}\right)-1$ is the background good rate of interest. But since $P_{t}^{y}=\beta^{t-1} P_{1}^{y}$ in any equilibrium, $\left(P_{t}^{y} / P_{t+1}^{y}\right)-1=(1 / \beta)-1$. Hence the interest rate in the model $^{29}$ is constant in any equilibrium, as we

${ }^{29}$ Since there is another good in model 2, there is a second interest rate. The rate of interest on $Z$ is zero if the good is stored and nonpositive in any case. 
assume in the text. In model 1 , this result occurs because a marginal rate of transformation is assumed to be constant; in model 2, it arises from an assumed constant marginal rate of substitution.

Any candidate for equilibrium must have the following properties: for $t=$ $1,2, \ldots, T, K_{t+1}^{y}=0, P_{t}^{y}=\beta^{t-1} P_{1}^{y}$, and $y_{t}>0$. Then (A.6) can be substituted into (A.5) to obtain:

$$
z_{t} \geqslant 0, \frac{f^{\prime}\left(z_{t}\right)}{a}-\frac{P_{t}^{z}}{P_{t}^{y}} \leqslant 0,
$$

with complementary slackness. This condition is referred to in the text as the consumer demand curve. Specifically, $f^{\prime}(0) / a$ is the choke price, $P_{t}^{z} / P_{t}^{y}$ is the price of the resource at time $t$, and $f^{\prime}\left(z_{t}\right) / a$ is the inverse demand curve. Note that assumptions about the demand curve made in the text are in fact implications of this fully specified model (as they are of model 1): the demand curve is time separable, stationary, dependent on the contemporaneous price of the resource and on no other prices, and independent of income or wealth.

Finally, we consider how to determine the equilibrium of this fully specified model. We can confine our search to cases where $K_{t+1}^{y}=0, P_{t}^{y}=\beta^{t-1} P_{1}^{y}$, and $y_{t}>0$, for $t=1,2, \ldots, T$. To determine the equilibrium sequence of prices $\left\{P_{t}^{z}\right\}$ we use the following facts. If $P_{t}^{z} / P_{t}^{y}<f^{\prime}(0) / a,\left(\mathrm{~A} .5^{\prime}\right)$ implies that $z_{t}>$ 0 . This demand for $Z$ must be satisfied by stored $Z$. If $Z$ is to be stored, the price of $Z$ must remain constant. From (A.7), for $t=1,2, \ldots, T$,

$$
K_{t+1}^{z} \geqslant 0, \frac{P_{t+1}^{z}}{P_{t+1}^{y}}-\frac{P_{t}^{z}}{P_{t+1}^{y}}=\frac{P_{t+1}^{z}}{P_{t+1}^{y}}-\frac{P_{t}^{z}}{\beta P_{t}^{y}} \geqslant 0,
$$

with complementary slackness. That is, if $Z$ is stored, its price (relative to the price of $Y$ ) must rise at the rate of interest for all $P_{t}^{z} / P_{t}^{y}<f^{\prime}(0) / a$. Finally, it can easily be shown that wealth maximization requires the cumulative sales of $Z$ to equal $\bar{K}^{z}$. What is referred to in the text as the equilibrium price path of the Hotelling model can be regarded as the sequence of relative prices $\left(P_{t}^{z} / P_{t}^{y}\right)$ that solves these conditions simultaneously.

The foregoing model generalizes easily to uncertainty and exhibits the martingale property which underlies the functional conditions of Section III. This is demonstrated in Samuelson (1971) and-in considerable detail-in Kohn (1978). A more comprehensive discussion of restrictions on general equilibrium models which yield the martingale property can be found in LeRoy (1982).

\section{Appendix B}

\section{Attack on Gold}

Between 1934 and 1968, the United States pegged the nominal price of gold at $\$ 35$ per ounce. Hence, the real price varied inversely with the price level. When speculators attacked in 1968, the government salvaged some of its buffer stock by closing the gold window. In the 1970s, it began to consider disposing of the portion retained.

For simplicity, we will assume that inflation proceeded each period at a constant rate $(\pi)$ so that the official price of gold-in real terms-declined geometrically. Furthermore, we will assume that speculators anticipated that the government would be unwilling to sell its entire stock $\left(G_{t}\right)$ in any period $t$ to defend the official price but would instead initially retain $\bar{G}$ for subsequent 
disposition. We will suppose speculators anticipated that the portion retained would be auctioned at some unknown time interval $(\tilde{t}=1,2, \ldots)$ after the rest of the government buffer stock was exhausted and that $\tilde{t}$ was taken to be a geometrically distributed, integer-valued random variable with mean $1 / \alpha$.

The government might not have behaved as the speculators anticipated. But simply because we must make some assumption about the relation of the prior expectations of the speculators to the subsequent conduct of the government-and no alternative assumptions seem superior-we will assume that speculators' expectations proved correct both about the amount of gold retained by the government and about the manner and stochastic timing of its subsequent disposition. ${ }^{30}$

We begin once again by considering the equilibrium in the absence of speculative purchases. Inflation would have progressively eroded the real official price-stimulating increasing demand-until the government stocks eventually dwindled to $\bar{G}$. At that point, the government would have suspended its pegging operation, and the real price would have soared high enough either to eliminate demand entirely or, alternatively, to provide enough incentive for foresighted extractors initially to have withheld some of their stocks from the government when the stockpile was first created in order to sell them after its exhaustion.

When speculative purchases are permitted, this price path ceases to be an equilibrium since it creates an incentive to purchase infinite stocks immediately before the upward jump in price. Such behavior would "tend" to shorten the duration of the pegging operation and reduce the market price which would prevail following its demise. In the new equilibrium, as soon as stocks fall below the attack threshold, speculators would purchase the remaining resources $(G-\bar{G})$, the government would close its window on $\bar{G}$ units, and there would be no upward jump in price. ${ }^{31}$

The attack threshold $\left(A^{+}\right)$may be determined as before by expressing the price immediately before and after the attack as functions of the government stock $(G)$ just prior to the attack and then finding that unique stock which equalizes the two prices.

Since the nominal price $\left(\bar{P}_{n}\right)$ is pegged in this case, the real official price $\left(\bar{P}_{t}\right)$ prior to the attack is no longer constant as was its counterpart in figure 2 . As time elapses, the stock held by the government $(G)$ falls from $\bar{K}$ and the real official price $(\bar{P})$ falls from $\bar{P}_{n}$. Hence the plot of $\bar{P}$ against $G$ (with time varied parametrically) in figure 10 is an upward-sloping curve passing through the point $\left(\bar{K}, \bar{P}_{n}\right){ }^{32}$

As before, the market price immediately following the attack $\left(P_{S H}\right)$ is a decreasing function of whatever stocks are held privately then $(K)$. But the

${ }^{30}$ If, e.g., speculators anticipated that the government would retain less than the government actually did retain following the attack, an upward jump in the market price above the real official price would occur.

${ }^{31} \mathrm{As}$ in the text, this assertion is accurate in continuous time but requires minor modification in discrete time because of an integer problem. If the speculative attack occurs at $t$, the market price on that period lies in the interval $\left[\bar{P}_{t}, \bar{P}_{t}(1+r)\right)$.

${ }^{32}$ Since $\bar{P}_{t}=\left[\bar{P}_{n} /(1+\pi)^{t-1}\right]$ and

$$
G_{t}=\bar{K}-\sum_{s=1}^{t-1} D\left[\bar{P}_{n} /(1+\pi)^{s-1}\right], t=1,2, \ldots,
$$

the government reserves and the real official price $\left(G_{t}, P_{t}\right)$ can be computed for each $t$. As time elapses, $G$ and $\bar{P}$ decline, respectively, from $\left(\bar{K}, P_{n}\right)$. Hence $d \bar{P} / d G>0$. 


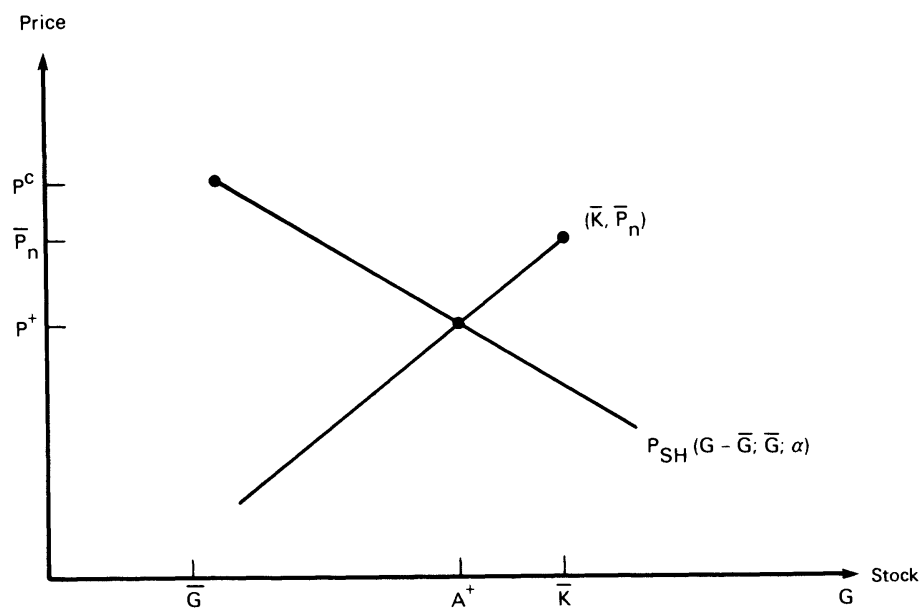

FIG. 10.-Determination of the attack threshold for gold when the nominal price is pegged, inflation persists, and the government is expected to retain temporarily part of its reserves.

market price would also be affected by two exogenous parameters-the amount retained by the government $(\bar{G})$ and the probability per period that it would be auctioned $(\alpha)$. Hence we denote $P_{S H}$ as follows: $P_{S H}=P_{S H}(K ; \bar{G} ; \alpha)$. In a previous paper, Salant and Henderson (1978, p. 634) showed how the initial price on an equilibrium path can be determined when there is a probability $\alpha$ that the government might auction $\bar{G}$ units of gold and speculators own a stock $(K)$ of any given size. Hence, the $P_{S H}$ function above is a reduced form of the Salant-Henderson gold model, just as the $P_{H}$ function of Section II is a reduced form of the Hotelling oil model. Following an attack in which the government retained $\bar{G}$ of its reserves $(G)$, the private market would acquire $G-\bar{G}$. In figure $10, P_{S H}(G-\bar{G} ; \bar{G} ; \alpha)$ is plotted as a function of $G$. The function is continuous and decreasing. When $G=\bar{G}, P_{S H}=P^{c}$.

The two curves in figure 10 intersect at the point $\left(A^{+}, P^{+}\right)$. The first coordinate indicates that the speculative attack occurs when the buffer stock declines below the attack threshold, $A^{+}(>\bar{G})$. At that point, speculators attack, the government salvages $\bar{G}$ for subsequent auction at an unknown date, and the price rises without a jump along the trajectory described in detail in Salant and Henderson (1978, pp. 635-36).

Changes in government policy parameters alter the date of the speculative attack. A decrease in the rate of inflation causes the upward-sloping curve in figure 10 to flatten and pivot around $\left(\bar{K}, \bar{P}_{n}\right)$ so that a smaller stock is associated with each real official price. ${ }^{33}$ The point of intersection shifts to the left,

${ }^{33}$ Pick any level for the real value of the official price. Let $t\left(t^{\prime}\right)$ denote the period in which that chosen level is reached if the nominal price is maintained at $\bar{P}_{n}$ and the inflation rate is $\pi\left(\pi^{\prime}\right)$. If $\pi^{\prime}<\pi, t^{\prime}>t$. Let $G_{t}\left(G_{t^{\prime}}^{\prime}\right)$ denote the remaining government stock when the official price has eroded to the specified level. In the text, it is claimed that $G_{t}>G_{t^{\prime}}^{\prime}$. This is equivalent to the claim that prior cumulative demand will be higher in the case with the lower inflation rate. This follows since (1) in each period before the official price reaches the specified level, demand would be greater in the case with the 
implying that the smaller the rate of inflation, the lower the attack threshold. Since it takes longer at higher real prices for the stock to be reduced to any given level, lowering the rate of inflation must also delay the speculative attack.

In Salant and Henderson (1978, p. 637) increases in the probability of an expected auction $(\alpha)$ are shown to depress the price. An increase in $\alpha$ would therefore shift the downward-sloping curve in figure 10 to the left so that the two curves intersected at a lower attack threshold. Hence, the shorter the expected interval between the date of the attack and the subsequent auction, the longer speculators would postpone the attack.

Finally, it can be shown that if a smaller portion of a given stock is in fact retained by the government for subsequent auction, the price will initially be lower: $d P_{S H} /\left.d \bar{G}\right|_{\alpha, G}=P_{S H, 2}-P_{S H, 1}>0$. Thus, if speculators anticipate that less will be withheld, the downward-sloping curve in figure 10 shifts to the left and the intersection occurs at a lower attack threshold-implying once again that the attack occurs later. Hence, if the government reduced the rate of inflation to zero and fostered the belief that the entire stockpile would be committed to the defense of the official price, the attack would be delayed the longest. But this is the case studied in Section II.

\section{References}

Bergsten, C. Fred. "The Purpose and Organization of Economic Stockpiling." Hearing before Joint Committee on Defense Production, 94th Cong., June $8,1976$.

- "The Policy of the United States toward International Commodity Agreements." Statement before Subcommittee on Economic Stabilization, House Committee on Banking, Finance and Urban Affairs, 95th Cong., June 8, 1977.

Blackwell, David. “Discounted Dynamic Programming.” Ann. Math. Statis. 36 (February 1965): 226-35.

Burke, William, and Levy, Y. Silver: End of an Era. Monthly Review Supplement. San Francisco: Federal Reserve Bank San Francisco, 1969.

Chung, K. L., and Fuchs, W. H. J. "On the Distribution of Values of Sums of Random Variables.” Memoirs American Math. Soc., no. 6 (1951), pp. 1-12.

Commodity Research Unit Limited. Co-ordinated National Stockpiles as a Market Stabilizing Mechanism: Copper. London: Commodity Res. Unit Ltd., 1977.

Dasgupta, Partha S., and Heal, Geoffrey M. Economic Theory and Exhaustible Resources. Cambridge: Cambridge Univ. Press, 1979.

Flood, Robert P., and Garber, Peter M. "A Systematic Banking Collapse in a Perfect Foresight World.” Working Paper no. 691. Cambridge, Mass.: Nat. Bur. Econ. Res., June 1981.

- "Gold Monetization and Gold Discipline." Mimeographed. Charlottesville: Univ. Virginia, 1982.

Fox, William. Tin: The Working of a Commodity Agreement. London: Mining J. Books, 1974.

lower inflation rate than it would be in the case with the higher inflation rate (since the real price would be lower); and (2) the path with the lower inflation rate requires longer $\left(t^{\prime}>t\right)$ to reach the specified level. 
Garber, Peter M. "The Lender of Last Resort and the Run on the Savings and Loans.” Working Paper no. 823. Cambridge, Mass.: Nat. Bur. Econ. Res., December 1981.

Gardner, Bruce L. Optimal Stockpiling of Grain. Lexington, Mass.: Lexington, 1979.

Goreux, L. M. “Optimal Rule of Buffer Stock Intervention.” Res. Dept. Memorandum no. DM/78/7. Washington: Internat. Monetary Fund, January 1978 .

Gustafson, Robert L. "Carry-Over Levels for Grains.” Tech. Bull. no. 1178. Washington: Dept. Agriculture, 1958.

Hotelling, Harold. "The Economics of Exhaustible Resources." J.P.E. 39, no. 2 (April 1931): 137-75.

Johnson, Harry G. "Commodities: Less Developed Countries' Demands and Developed Countries' Response.” In The New International Economic Order: The North-South Dialogue, edited by Jagdish N. Bhagwati. Cambridge, Mass.: MIT Press, 1977.

Kelley, John L. General Topology. Princeton, N.J.: Van Nostrand, 1955.

Kohn, Meier. "Competitive Speculation." Econometrica 46, no. 5 (September 1978): 1061-76.

Kolmogorov, A. N., and Fomin, F. V. Elements of the Theory of Functions and Functional Analysis: Metric and Normal Spaces. Rochester, N.Y.: Graylock, 1957.

Koromzay, Val. "A Convenience Yield Model of Speculative Attack in Foreign Exchange Markets.” Unpublished draft, 1978.

Krugman, Paul R. "A Model of Balance-of-Payments Crises." J. Money, Credit and Banking 11 (August 1979): 311-25.

LeRoy, Stephen F. "Expectations Models of Asset Prices: A Survey of Theory." J. Finance 37 (March 1982): 185-217.

Lucas, Robert E., Jr. "Econometric Policy Evaluation: A Critique." CarnegieRochester Conference Series on Public Policy. J. Monetary Econ. 1, suppl. (1976): 19-46.

Miller, Ross M.; Plott, Charles R.; and Smith, Vernon L. "Intertemporal Competitive Equilibrium: An Empirical Study of Speculation." Q.J.E. 91 (November 1977): 599-624.

Salant, Stephen W., and Henderson, Dale W. "Market Anticipations of Government Policies and the Price of Gold.” J.P.E. 86, no. 4 (August 1978): $627-48$.

Samuelson, Paul A. "Stochastic Speculative Price." Proc. Nat. Acad. Sci. USA 68 (1971): 335-37.

Schink, George R., and Smith, Gordon W. "The International Tin Agreement: A Reassessment." Econ. J. 86 (December 1976): 715-28.

Townsend, Robert M. "The Eventual Failure of Price Fixing Schemes." J. Econ. Theory 14 (February 1977): 190-99.

Weinstein, Milton C., and Zeckhauser, Richard J. "The Optimal Consumption of Depletable Natural Resources." Q.J.E. 89 (August 1975): 371-92.

Wenocur, R. D. S., and Salant, Stephen W. "Recurrence of a Modified Random Walk and Its Application to an Economic Model." SIAM J. Appl. Math. 40 (February 1981): 163-66. 
http://www.jstor.org

\title{
LINKED CITATIONS
}

\author{
- Page 1 of 2 -
}

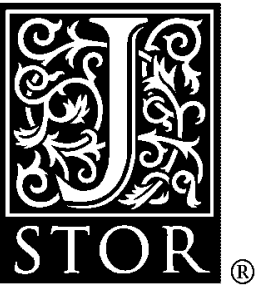

You have printed the following article:

The Vulnerability of Price Stabilization Schemes to Speculative Attack

Stephen W. Salant

The Journal of Political Economy, Vol. 91, No. 1. (Feb., 1983), pp. 1-38.

Stable URL:

http://links.jstor.org/sici?sici=0022-3808\%28198302\%2991\%3A1\%3C1\%3ATVOPSS\%3E2.0.CO\%3B2-Q

This article references the following linked citations. If you are trying to access articles from an off-campus location, you may be required to first logon via your library web site to access JSTOR. Please visit your library's website or contact a librarian to learn about options for remote access to JSTOR.

\section{References}

\section{Competitive Speculation}

Meir Kohn

Econometrica, Vol. 46, No. 5. (Sep., 1978), pp. 1061-1076.

Stable URL:

http://links.jstor.org/sici?sici=0012-9682\%28197809\%2946\%3A5\%3C1061\%3ACS\%3E2.0.CO\%3B2-Z

\author{
A Model of Balance-of-Payments Crises \\ Paul Krugman \\ Journal of Money, Credit and Banking, Vol. 11, No. 3. (Aug., 1979), pp. 311-325. \\ Stable URL: \\ http://links.jstor.org/sici?sici=0022-2879\%28197908\%2911\%3A3\%3C311\%3AAMOBC\%3E2.0.CO\%3B2-6
}

\section{Expectations Models of Asset Prices: A Survey of Theory}

Stephen F. Leroy

The Journal of Finance, Vol. 37, No. 1. (Mar., 1982), pp. 185-217.

Stable URL:

http://links.jstor.org/sici?sici=0022-1082\%28198203\%2937\%3A1\%3C185\%3AEMOAPA\%3E2.0.CO\%3B2-1

\section{Stochastic Speculative Price}

Paul A. Samuelson

Proceedings of the National Academy of Sciences of the United States of America, Vol. 68, No. 2.

(Feb., 1971), pp. 335-337.

Stable URL:

http://links.jstor.org/sici?sici=0027-8424\%28197102\%2968\%3A2\%3C335\%3ASSP\%3E2.0.CO\%3B2-P 
http://www.jstor.org

\section{LINKED CITATIONS \\ - Page 2 of 2 -}

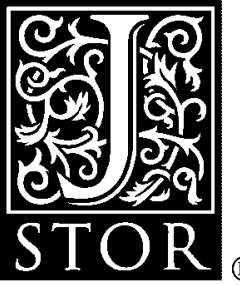

Recurrence of a Modified Random Walk and its Application to an Economic Model

Roberta S. Wenocur; Stephen W. Salant

SIAM Journal on Applied Mathematics, Vol. 40, No. 1. (Feb., 1981), pp. 163-166.

Stable URL:

http://links.jstor.org/sici?sici=0036-1399\%28198102\%2940\%3A1\%3C163\%3AROAMRW\%3E2.0.CO\%3B2-W 